\title{
Processo de exclusão simples com taxas variáveis
}

\author{
Adriana Uquillas Andrade
}

\author{
TESE APRESENTADA \\ Instituto de MATEMÁtica E Estatística \\ UNIVERSIDADE DE SÃo PAULO \\ PARA \\ OBTENÇÃAO DO TÍTULO \\ DOUTOR EM CIÊNCIAS
}

\author{
Área de Concentração: Estatística \\ Orientador: Prof. Dr. Adilson Simonis
}

Durante o desenvolvimento deste trabalho o autor recebeu auxílio financeiro da CAPES

São Paulo, junho de 2008 


\section{Processo de exclusão simples com taxas variáveis}

Este exemplar corresponde à redação final da tese devidamente corrigida e defendida por Adriana Uquillas Andrade

e aprovada pela Comissão Julgadora.

Banca Examinadora:

- Prof. Dr. Adilson Simonis - IME/USP.

- Prof. Dr. Claudio Landim - IMPA.

- Prof. Dr. Pablo Ferrari - IME/USP.

- Profa.Dra.Nancy Lopes Garcia-IMECC/UNICAMP.

- Prof.Dr. Rafael A. Rosales Mitowsky -FFCLRP/USP. 


\section{Agradecimentos}

Ao meu orientador Adilson Simonis pelo tempo e apoio na realização deste trabalho, e especialmente pela sua amizade.

Ao Professor Claudio Landim por ter reservado tempo e dedição inestimáveis para o desenvolvimento deste trabalho.

Ao Professor Pablo Ferrari pela atenção que recebi dele sempre que precisei.

Aos meus pais, Elena e Roberto, pelo amor, apoio e força que recebi deles durante o desenvolvimento deste trabalho.

Aos meus irmãos, pelo exemplo que sempre me deram. 


\section{Resumo}

Nosso trabalho considera o processo de exclusão simples do vizinho mais próximo evoluindo com taxas de salto aleatórias $\beta=\left\{\beta_{x}: x \in \mathbb{Z}\right\}$. Demonstramos o limite hidrodinâmico deste processo. Este resultado é obtido através do limite hidrodinâmico do processo de exclusão onde as taxas de salto $\left\{\beta_{x}: x \in \mathbb{Z}\right\}$ são substituidas pelas taxas $\left\{c_{x, N}: x \in \mathbb{Z}\right\}$ que tem a mesma distribuição que $\left\{\beta_{x}: x \in \mathbb{Z}\right\}$ para cada $N \geq 1$.

Fazemos algumas suposições no meio $c_{N}$ e consideramos que as partículas estão inicialmente distribuidas de acordo à medida produto de Bernoulli associada a um perfil inicial $\rho_{0}: \mathbb{R} \rightarrow[0,1]$.

Palavras-chave: limite hidrodinâmico, meio aleatório, sistemas de partículas. 


\section{Abstract}

Consider a Poisson process with rate equal to 1 in $\mathbb{R}$. Consider the nearest neighbor simple exclusion process with random jump rates $\beta=\left\{\beta_{x}: x \in \mathbb{Z}\right\}$, where $\beta_{x}=\lambda, \lambda>0$ if there is a Poisson mark between $(x, x+1)$ and $\beta_{x}=1$ otherwise. We prove the hydrodynamic limit of this process. This result follows from the hydrodynamic limit in the case that the jump rates $\left\{\beta_{x}: x \in \mathbb{Z}\right\}$ are replaced by an array $\left\{c_{x, N}: x \in \mathbb{Z}\right\}$ having the same distribution for each $N \geq 1$.

Keywords: hydrodynamic limit, random environment, interacting particle system. 


\section{Conteúdo}

1 Introdução $\quad 1$

2 Limite Hidrodinâmico $\quad 5$

$2.1 \quad$ O Processo $Z\left(t \mid u, v_{E}\right) \ldots \ldots \ldots \ldots \ldots \ldots \ldots \ldots \ldots$

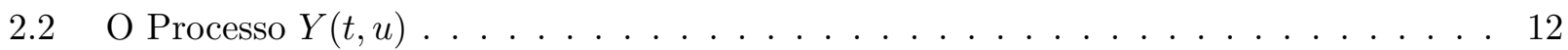

2.3 Estudo do Limite Hidrodinâmico . . . . . . . . . . . . . . . . . . . . . 18

3 Conglomerados $\quad 33$

3.1 Demonstrações das proposições $\ldots \ldots \ldots \ldots$. . . . . . . . . . . . . 39 


\section{Capítulo 1}

\section{Introdução}

Passeios aleatórios em meios aleatórios tem sido muito estudado nos últimos anos. Nosso trabalho considera o processo de exclusão simples do vizinho mais próximo evoluindo num meio aleatório que chamaremos de $\beta$. Isto é:

Considere um processo de Poisson com taxa 1 na reta $\mathbb{R}$. Vamos denotar suas marcas por $\ldots, \gamma_{-n}<\gamma_{-n+1}<\ldots<\gamma_{-1}<0<\gamma_{0}<\gamma_{1}<\ldots$

Considere o processo de exclusão simples com taxas $\beta=\left\{\beta_{x}: x \in \mathbb{Z}\right\}$, onde $\beta_{x}=\lambda, \lambda>0$ se existir uma marca de Poisson entre $(x, x+1)$ e $\beta_{x}=1$ caso contrário. Denotamos por $\eta$ as configurações em $\{0,1\}^{\boldsymbol{Z}}$ tais que $\eta(x)=1$ se o sítio $x$ está ocupado e $\eta(x)=0$ caso contrário. Com taxa $\beta_{x}$ os valores das variáveis $\eta(x)$ e $\eta(x+1)$ são intercambiados.

Dada uma função $f$ cilíndrica e um campo aleatório $\beta=\left\{\beta_{x}: x \in \mathbb{Z}\right\}$, o gerador $L$ do processo pode ser escrito como:

$$
L f(\eta)=\sum_{x \in \mathbb{Z}} \beta_{x}\left\{f\left(\sigma^{x, x+1} \eta\right)-f(\eta)\right\}
$$

onde $\sigma^{x, x+1} \eta$ é a configuração obtida de $\eta$ ao intercambiar as variáveis $\eta(x)$ e $\eta(x+1)$, isto é, 


$$
\sigma^{x, x+1} \eta(y)= \begin{cases}\eta(x+1) & \text { se } y=x \\ \eta(x) & \text { se } y=x+1 \\ \eta(y) & \text { caso contrario }\end{cases}
$$

O resultado principal neste trabalho consiste na demonstração do limite hidrodinâmico deste processo.Enunciaremos este resultado na sequência.

Vamos substituir as taxas de salto $\left\{\beta_{x}: x \in \mathbb{Z}\right\}$ pelas taxas $c_{N}=\left\{c_{x, N}: x \in \mathbb{Z}\right\}, N \geq 1$ que tem a mesma distribuição que $\left\{\beta_{x}: x \in \mathbb{Z}\right\}$ para cada $N \geq 1$, e a partir do limite hidrodinâmico obtido para este processo vamos obter o limite hidrodinámico "annealed"para o processo de exclusão em $\{0,1\}^{\mathbb{Z}}$ tal que com taxa $\beta_{x}$ os valores das variáveis $\eta(x)$ e $\eta(x+1)$ são intercambiados.

A seqüência $\left\{c_{x, N}: x \in \mathbb{Z}\right\}$ é construida da seguinte maneira:

$$
c_{y, N}=\frac{1}{N\left\{W\left(\frac{y+1}{N}\right)-W\left(\frac{y}{N}\right)\right\}}
$$

onde $W: \mathbb{R} \rightarrow \mathbb{R}$ é definida como

$$
W\left(\frac{k}{N}\right)=\frac{k}{N}+\sigma(k)\left(\frac{1}{\lambda}-\frac{1}{N}\right) \sum_{j=0}^{\infty} \mathbf{1}_{\left\{\gamma_{j} \in[0, k / N]\right\}}
$$

e $\sigma(k)=1$ se $k \geq 0$ e $\sigma(k)=-1$ se $k<0$.

Assim, a seqüência $\left\{c_{x, N}: x \in \mathbb{Z}\right\}$ definida como função de $W$ tem a mesma distribuição de $\left\{\beta_{x}: x \in \mathbb{Z}\right\}$.

Portanto, o processo que iremos estudar se comporta da seguinte maneira: Seja $x \in \mathbb{Z}$ e $N$ inteiro positivo, consideramos o processo de exclusão simples do vizinho mais próximo onde uma partícula que está em $x$ (respectivamente $x+1$ ) vai para $x+1$ (respectivamente $x$ ) com taxa $c_{x, N}=N^{2}$ se não houver marca de Poisson entre os sítios $x$ e $x+1$, e caso contrário salta com taxa $c_{x, N}=\lambda N$, para algum $\lambda>0$.

Sob algumas suposições no meio $c_{N}$ provamos que se as partículas estão inicialmente distribuidas de acordo com a medida produto de Bernoulli associada ao perfil inicial $\rho_{0}: \mathbb{R} \rightarrow[0,1]$, então o perfil de densidade, $\rho=\rho(t, x)$, evolui como a solução da equação de difusão com fronteiras

$$
\begin{cases}\partial_{t} \rho & =\Delta \rho \text { nos intervalos }\left(\gamma_{j}, \gamma_{j+1}\right) \\ \partial_{x} \rho\left(t, \gamma_{j}+\right) & =\partial_{x} \rho\left(t, \gamma_{j}-\right) \\ \partial_{x} \rho\left(t, \gamma_{j}+\right) & =\lambda\left[\rho\left(t, \gamma_{j}-\right)-\rho\left(t, \gamma_{j}+\right)\right]\end{cases}
$$


onde $\Delta \rho$ é o Laplaciano de $\rho$.

Este resultado permite, entre outras coisas, uma aplicação no estudo da formação de conglomerados no nosso processo. Especificamente, apresentamos uma cota superior e uma inferior para a distribuição do que chamamos de tempo de escape da partícula na origem. Mais precisamente, dado que consideramos um perfil inicial onde, com probabilidades altas as partículas são posicionadas nos sítios $-j,-j+1, \ldots, j-1, j, j$ inteiro positivo e com probabilidades próximas de zero fora desses sítios, determinamos uma estimativa da distribuição do tempo em que a partícula que está inicialmente na origem consegue se movimentar pela primeira vez.

Alguns resultados existentes na literatura serão necessários para o desenvolvimento de nosso trabalho, que serão referenciados ao longo do texto como Resultados, em quanto que as nossas contribuições serão referenciadas como Lemas, Proposições e Teoremas. 


\section{Capítulo 2}

\section{Limite Hidrodinâmico}

Considere o espaço quociente $\Pi_{N}=\mathbb{Z} / N \mathbb{Z}$, onde $N$ é um inteiro positivo. Os pontos de $\Pi_{N}$ serão representados pelas letras $x, y, z$. Considere um processo de Poisson com taxa 1 na reta $\mathbb{R}$. Vamos denotar suas marcas por $\ldots, \gamma_{-n}<\gamma_{-n+1}<\ldots<\gamma_{-1}<0<\gamma_{0}<\gamma_{1}<\ldots$.

A dinâmica que iremos estudar é um processo de exclusão simples evoluindo em $\mathbb{Z}$ que pode ser descrito informalmente como segue. Denote por $\eta$ as configurações de $\{0,1\}^{\boldsymbol{Z}}$ tais que $\eta(x)=1$ se o sítio $x$ está ocupado e $\eta(x)=0$ caso contrário. Se não existir uma marca de Poisson entre os sítios $\frac{x}{N}$ e $\frac{x+1}{N}$ a partícula se comporta como um processo de exclusão simples simétrico evoluindo com taxa $N^{2}$; caso contrário, com taxa $\lambda N, \lambda>0$, os valores das variáveis $\eta(x)$ e $\eta(x+1)$ são intercambiados. O principal objetivo é descrever o limite hidrodinâmico do processo.

Utilizaremos as seguintes notações: dado um intervalo $I \subset \mathbb{R}, D(I, \mathbb{R})$ indicará o espaço das funções $f: I \rightarrow \mathbb{R}$ tais que os limites laterais $f(x+)$ e $f(x-)$ existem e $f(x+)=f(x)$, para cada $x \in I$, munido com a métrica de Skorohod (veja definição a seguir); estas funções são importantes no estudo de processos estocásticos que admitem ou requerem saltos. $\mathbb{D}(f)$ indicará o conjunto das discontinuidades de uma função $f: I \rightarrow \mathbb{R}$ e $f^{-1}$ indicará a inversa de $f$, definida por

$$
f^{-1}(y)=\sup \{x \in I: f(x) \leq y\}
$$

Finalmente, para $x, y \in \mathbb{R}$, o máximo e o mínimo entre $x$ e $y$ serão indicados por $x \vee y:=$ $\max \{x, y\}$ e $x \wedge y:=\min \{x, y\}$.

A métrica de Skorohod $d_{S}$ em $D(I, \mathbb{R})$ é apresentada na seguinte definição: 
Definição 1 Para $f, g \in D(I, \mathbb{R})$, a métrica no espaço $D(I, \mathbb{R})$ é dada por

$$
d_{S}(f, g)=\inf _{\kappa}\left[\phi(\kappa) \vee \int_{0}^{\infty} e^{-u} d(f, g, \kappa, u) d u\right],
$$

onde

$$
\phi(\alpha)=\sup _{s>t \geq 0}\left|\log \frac{\alpha(s)-\alpha(t)}{s-t}\right|<\infty
$$

$e$

$$
d(f, g, \alpha, u)=\sup _{t \geq 0} q(f(t \wedge u), g(\alpha(t) \wedge u))
$$

com $\alpha:[0, \infty) \rightarrow[0, \infty)$ função (estritamente) crescente e q a métrica definida por $q(x, y)=\min \{\mid x-$ $y \mid, 1\}$.

Da definição acima temos que duas trajetórias na topologia de Skorohod estão próximas se em qualquer intervalo de tempo limitado, estas trajetórias estão uniformemente próximas depois de uma pequena distorção $\alpha$ no eixo do tempo. No subespaço de funções contínuas, convergência na métrica $d_{S}$, é o mesmo que convergência uniforme nos intervalos compactos.

Definição 2 Considere um movimento Browniano $B(t, \omega)$ definido num espaço de probabilidade $(\mathcal{X}, \mathcal{B}, \mathbb{P})$. Definimos o tempo local $L: \mathbb{R}^{+} \times \mathbb{R} \rightarrow \mathbb{R}^{+}$por

$$
\int_{0}^{t} \mathbf{1}\{B(s, .) \in A\} d s=\int_{A} L(t, x) d x
$$

para todo conjunto de Borel $A \subset(-\infty, \infty)$ e todo $t \geq 0$. Isto é, o tempo local é uma medida de quanto tempo $B(s,$.$) permanece no conjunto A$ no intervalo de tempo $[0, t]$

Por simplicidade, escreveremos $B(t, \omega)=B(t)$. Para todos os processos definidos na seqüência deste trabalho, que são função do movimento Browniano $B(t)$, usaremos esta mesma notação.

Considere a função de salto $W: \mathbb{R} \rightarrow \mathbb{R}$ definida por

$$
W\left(\frac{k}{N}\right)=\frac{k}{N}+\sigma(k)\left(\frac{1}{\lambda}-\frac{1}{N}\right) \sum_{j=0}^{\infty} \mathbf{1}_{\left\{\gamma_{j} \in[0, k / N]\right\}}
$$

onde $\sigma(k)=1$ se $k>0$ e $\sigma(k)=-1$ caso contrario. 
Fixe $N \geq 1, x \in \mathbb{Z}$ e uma realização de $W$. Considere o passeio aleatório $X_{N}(t, x)$ definido nos inteiros começando no ponto $x$ que salta de $x$ a $x+1$ e de $x+1$ a $x$ com taxa $N^{2}$ se não houver uma marca de Poisson entre $x$ e $x+1$. Caso contrário salta com taxa $\lambda N$. O gerador deste processo, para $f$ cilíndrica, é dado por

$$
\left(\mathbb{L}_{N} f\right)(x / N)=N^{2} c_{x, N}\{f(x+1)-f(x)\}+N^{2} c_{x-1, N}\{f(x-1)-f(x)\}
$$

onde

$$
c_{y, N}=\frac{1}{N\left\{W\left(\frac{y+1}{N}\right)-W\left(\frac{y}{N}\right)\right\}} .
$$

Por simplicidade omitiremos a dependência de $\mathrm{N}$ em $c_{y, N}$ e escreveremos $c_{y}$.

Vamos descrever o comportamento assintótico deste passeio aleatório e, para isto, alguns resultados obtidos por Stone [16] (1962) serão úteis:

Seja $v$ a medida de Lebesgue em $\mathbb{R}$. Considere a medida $v \mathbf{1}\{E\}:=v_{E}$, para $E$ de Borel, onde $E^{C}=\left\{\left(W\left(\frac{x-}{N}\right), W\left(\frac{x}{N}\right)\right):\right.$ existe pelo menos

$$
\text { uma marca de Poisson no elo } \left.\left(\frac{x-1}{N}, \frac{x}{N}\right)\right\} \text {. }
$$

A medida $v_{E}$ tem suporte, denotado por $\operatorname{supp}\left(v_{E}\right)$, dado por

$$
\operatorname{supp}\left(v_{E}\right)=\{W(x), W(x-): x \in \mathbb{R}\}
$$

onde $W(x-):=\lim _{y \rightarrow x-} W(y)$. Para cada $u \in \operatorname{supp}\left(v_{E}\right)$ e $t \geq 0$ definimos

$$
\begin{gathered}
\varphi\left(t \mid u, v_{E}\right)=\int_{\mathbb{R}} L(t, y-u) v_{E}(d y), \\
\varphi^{-1}\left(t \mid u, v_{E}\right)=\sup \left\{s \geq 0: \varphi\left(s \mid u, v_{E}\right) \leq t\right\}
\end{gathered}
$$

$\mathrm{e}$

$$
Z\left(t \mid u, v_{E}\right)=B\left(\varphi^{-1}\left(t \mid u, v_{E}\right)\right)+u
$$

Observação: Para toda função $f$ contínua em $\mathbb{R}$ com suporte compacto, temos

$$
\int_{a}^{b} f(u) v_{E}(d u)=\int_{W^{-1}(a)}^{W^{-1}(b)} f(W(x)) d x .
$$


Considere as seguintes definições

Definição 3 Seja $X_{t}(f)=f(t)$ para $f \in D([0, \infty), I M)$, onde $I M$ é um espaço métrico separável. Considere o processo de Markov $P_{x}$, onde $\left\{P_{x}: x \in \mathbb{M}\right\}$ são as medidas de probabilidade no espaço $D([0, \infty), M)$. Para $g$ mensurável e limitada em $\mathbb{M}$, seja $S(t) g(x)=\mathbb{E}^{x} g\left(X_{t}\right)$. O processo $P_{x} e^{\prime}$ dito processo de Feller se $S(t) g \in C_{b}(I M)$, para todo $t \geq 0$ e $f \in C_{b}(I M)$.

Definição 4 Considere o processo de Feller $P_{x}$ definido acima. Vamos definir a propiedade forte de Markov. Seja $\tau: D([0, \infty), M) \rightarrow[0, \infty]$ um tempo de parada com respeito à filtração $\mathcal{F}_{t}=\sigma\left(X_{s}:\right.$ $s \leq t)$, e defina $\theta_{\tau} f(x):=f(\tau(f)+x)$. $P_{x}$ é fortemente Markov se e somente se

$$
P_{x}\left[\theta_{\tau}^{-1} A \mid \mathcal{F}_{\tau}\right](f)=P_{f(\tau)}(A)
$$

para $P_{x}$-quase toda $f$ tal que $\tau(f)<\infty$, para todo $x \in I M$ e $A \in \mathcal{F}$.

Temos que, para cada $u \in \operatorname{supp}\left(v_{E}\right), \varphi\left(t \mid u, v_{E}\right)$ é não decrescente e contínua em $t$, estritamente positiva para $t>0$. A função $\varphi^{-1}$ é uma função não decresecente contínua à direita com limite à esquerda e $\varphi^{-1}(0, u)=0$. Como função de $t$ o processo $Z=\left\{Z\left(t \mid u, v_{E}\right): t \geq 0\right\}$ é contínuo à direita com limite à esquerda, definido no mesmo espaço do movimento Browniano $B(t)$, o espaço $\left(\operatorname{supp}\left(v_{E}\right), \mathcal{B}, \mathbb{P}\right)$. Além disso $Z$ é um processo forte de Markov com espaço de estados supp $\left(v_{E}\right)$ e estado inicial $u$. Como o $\operatorname{supp}\left(v_{E}\right)$ é um conjunto enumerável, então $Z$ é um processo de nascimento e morte.

Para $N \geq 1$, seja $v_{N}=\frac{1}{N} \sum_{x \in \mathbb{Z}} \delta_{W(x / N)} ; v_{N}$ coloca uma massa de $\frac{1}{N}$ em cada ponto $W(x / N)$. Observe que $v_{N}$ converge fracamente para $v_{E}$, pois $\lim _{N \rightarrow \infty} \int f d v_{N}=\int f d v \mathbf{1}\{E\}$.

Como conseqüência dos resultados de Stone (1963)[16] temos que se $\left\{u_{i}\right\}_{i \in \boldsymbol{Z}}$ é uma seqüência de números reais estritamente crescente tal que $\lim _{i \rightarrow \pm \infty} u_{i}= \pm \infty$ e definimos $v=\sum_{i \in \mathbb{Z}} w_{i} \delta_{u_{i}}$ onde $\left\{w_{i}\right\}_{i \in \mathbb{Z}}$ são pesos fixados. Então $Z\left(. \mid u_{j}, v\right)$ é um passeio aleatório a tempo contínuo em $\left\{u_{i}\right\}_{i \in \mathbb{Z}}$ começando em $u_{j}$, e dado que está na posição $u_{i}$, salta para $u_{i-1}, u_{i+1}$, respectivamente, com taxas

$$
\frac{1}{w_{i}\left(u_{i}-u_{i-1}\right)} \text { e } \frac{1}{w_{i}\left(u_{i+1}-u_{i}\right)}
$$

Pela definição de $v_{N}$ e pelo resultado, acima o passeio aleatório $X_{N}(., x) / N$ tem a mesma lei que o processo $W^{-1}\left(Z\left(. \mid W(x / N), v_{N}\right)\right)$. 
Para todo $x \in \mathbb{R}$, seja

$$
Y_{N}(t, x)=W^{-1}\left(Z\left(t \mid W\left(\frac{\lceil x N\rceil}{N}\right), v_{N}\right)\right)
$$

onde $\lceil x N\rceil$ é o maior inteiro menor ou igual a $x N$. Segue que

$$
\frac{X_{N}(.,\lceil x N\rceil)}{N} \text { tem a mesma lei que } Y_{N}(., x)
$$

para todo $N \geq 1$.

Observação: Uma medida $\mu$ é dita uma medida de Radon se todo ponto do espaço da medida tem uma vizinhança com medida $\mu$ finita, e se a medida do conjunto $A, \mu(A)$, pode ser aproximada por subconjuntos compactos mensuráveis $\left\{K_{n}: n \geq 1\right\}$. Isto é : $\mu(A)=\sup \left\{\mu\left(K_{n}\right) \mid K_{n} \subseteq\right.$ A compactos\}.

O seguinte resultado foi obtido por Stone [16] (1962)

Resultado 1 Seja $\left\{\mu_{n}\right\}_{n \geq 0}$ uma seqüência de medidas e $\mu$ uma medida de Radon em $\mathbb{R}$ com suporte não limitado nem por baixo nem por cima, tais que

- $\mu_{n} \rightarrow \mu$ fracamente

- se $y_{n} \in \operatorname{supp}\left(\mu_{n}\right)$ é uma seqüência convergente quando $n \uparrow \infty$, então $\lim _{n \uparrow \infty} y_{n} \in \operatorname{supp}(\mu)$

Seja $x_{n} \in \operatorname{supp}(\mu)$ uma seqüência convergente com $\lim _{n \uparrow \infty} x_{n}=x$. Então

$$
\lim _{n \uparrow \infty} d_{S}\left(Z\left(. \mid x_{n}, \mu_{n}\right), Z(. \mid x, \mu)\right)=0 \quad \mu q . c
$$

Temos que $\operatorname{supp}\left(v_{N}\right)$ consiste dos valores de $W(x / N)$. Seja $y_{N}=W(\lceil u N\rceil / N)$. Como $W(\lceil u N\rceil / N) \rightarrow$ $W(u)$ na métrica $d_{S}$ quando $N \rightarrow \infty$; existe uma seqüência de funções contínuas $h_{N}: \mathbb{R} \rightarrow \mathbb{R}$ tal que, para todo conjunto $K \subset \mathbb{R}$ compacto, temos:

$$
\lim _{N \rightarrow \infty} \sup _{u \in K}\left|u-h_{N}(u)\right|=0
$$

$\mathrm{e}$

$$
\lim _{N \rightarrow \infty} \sup _{u \in K} \mid W(\lceil u N\rceil / N)-W\left(h_{N}(u)\right)=0 .
$$


Isto implica que $y_{N} \rightarrow y$, onde $y=W(u)$ ou $W(u-)$, e portanto $y \in \operatorname{supp}\left(v_{E}\right)$.

Dado que $\lim _{N \uparrow \infty} W(\lceil x N\rceil / N)=W(x)$ e que $v_{N}$ converge fracamente para $v_{E}$, segue do Resultado 1 que, com probabilidade 1 ,

$$
\lim _{N \uparrow \infty} d_{S}\left(Z\left(. \mid W(\lceil x N\rceil / N), v_{n}\right), Z(. \mid W(x), v \mathbf{1}\{E\})\right)=0 .
$$

Considere agora o processo $Y(t, x)$, com $t \geq 0$ e $x \in \mathbb{R}$ definido por:

$$
Y(t, x)=W^{-1}(Z(t \mid W(x), v \mathbf{1}\{E\})) .
$$

Segue também de [15] que para todo $x \in \mathbb{R}$, com probabilidade 1 ,

$$
\lim _{N \rightarrow \infty} d_{S}\left(Y_{N}(., x)-Y(., x)\right)=0 .
$$

Além disso, $Y(., x)$ tem trajetórias contínuas quase certamente e, para todo $x \in \mathbb{R}$ e $T>0$, com probabilidade 1 , temos

$$
\lim _{N \rightarrow \infty} \sup _{0 \leq t \leq T}\left|Y_{N}(t, x)-Y(t, x)\right|=0 .
$$

\section{$2.1 \quad$ O Processo $Z\left(t \mid u, v_{E}\right)$}

Como definido anteriormente,

$$
Z\left(t \mid u, v_{E}\right)=B\left(\varphi^{-1}\left(t, u, v_{E}\right)\right)+u
$$

tem espaço de estados $\operatorname{supp}\left(v_{E}\right)=\{W(x), W(x-): x \in \mathbb{R}\}$. Um dos objetivos aqui é descrever como são as funções que pertencem ao domínio $\mathcal{D}_{W}^{Z}$ do gerador do processo $Z$. Para isto vamos descrever o gerador $\mathcal{L}_{W}^{Z}$ do processo $Z$ :

Seja $\{S(t): t \geq 0\}$ o semigrupo de Markov associado a $Z\left(t \mid u, v_{E}\right)$, isto é, seja

$$
S(t) f(u)=\mathbb{E}\left[f\left(Z\left(t \mid u, v_{E}\right)\right)\right]
$$

para $f$ função real, contínua, limitada em $\{W(x), W(x-): x \in \mathbb{R}\}$ e tal que, para todo $\epsilon>0$, a função $f$ tem módulo menor que $\epsilon$ fora de um subconjunto limitado $F$ do espaço de estados de $Z(t, u, v \mathbf{1}\{E\})$. 
Vamos denotar por

$C(A)$ ao espaço de funções reais contínuas em $A$,

$C_{b}(A)$ o espaço de funções reais contínuas e limitadas em $A$,

$C_{c}(A)$ o espaço de funções reais contínuas em $A$ com suporte compacto e

$C_{0}(A)$ o espaço de funções reais contínuas, limitadas em $A$, tais que para todo $\epsilon>0$ a funções tenham módulo menor que $\epsilon$ fora de um subconjunto limitado $F \subset A$ (funções que se anulam no infinito).

Considere a função de densidade de transição $p$ do Processo de Markov $Z\left(t \mid u, v_{E}\right)$ definida em $(0, \infty) \times \operatorname{supp}\left(v_{E}\right)$. A seguir, coletamos alguns resultados relacionados com processos de Markov, que serão utilizados na sequência. A demonstração desses resultados pode ser vista em Petr Mandl (1968) [17].

Para $u \in \operatorname{supp}\left(v_{E}\right)$ e $\delta>0, p$ satisfaz as seguintes propriedades:

1. $\lim _{t \rightarrow 0} t^{-1}\left[1-\int_{|u-w|<\delta} p(t, u, w) v_{E}(d w)\right]=0$

2. $\lim _{t \rightarrow 0} t^{-1} \int_{|u-w|<\delta}(w-u) p(t, u, w) v_{E}(d w)=b(u)$

3. $\lim _{t \rightarrow 0} t^{-1} \int_{|u-w|<\delta}(u-w)^{2} p(t, u, w) v_{E}(d w)=2 a(u)$

4. Para toda função $f \in C_{0}\left(\operatorname{supp}\left(v_{E}\right)\right)$ e com segunda derivada contínua,

$$
\lim _{t \rightarrow 0} t^{-1}\left[\int f(w) p(t, u, w) v \mathbf{1}\{E\}(d w)-f(u)\right]=a(u) f^{\prime \prime}(u)+b(u) f^{\prime}(u),
$$

onde $a$ e $b$ são os coeficientes de difusão e o drift, respectivamente.

Comparando a definição de gerador com o item 4 acima, tem-se que, num processo satisfazendo as condições 1,2 , e 3 , existe uma estreita relação entre o gerador $\mathcal{L}_{W}^{Z}$ e o operador diferencial

$$
a(u) \frac{d^{2}}{d u^{2}}+b(u) \frac{d}{d u}=\frac{d}{d v_{E}} \frac{d}{d u} .
$$


Sejam $f, g \in C_{0}\left(\operatorname{supp}\left(v_{E}\right)\right)$ tais que

$$
g(u)=\frac{d}{d v_{E}} \frac{d}{d u} f(u)=\mathcal{L}_{W}^{Z} f(u) .
$$

Então,

$$
f(u)=\int_{0}^{u} \int_{0}^{w} g(a) v_{E}(d a) d w+f(0)+u f^{\prime}(0) .
$$

Segue que

$$
f(u)=\int_{0}^{u} \int_{0}^{w} g(a) v_{E}(d a) d w+c+u e,
$$

onde na equação acima $c=f(0)$ e $e=f^{\prime}(0)$. Assim, o domínio do gerador do processo $Z$, denotado por $\mathcal{D}_{W}^{Z}$, é o conjunto de funções $f \in C_{0}\left(\operatorname{supp}\left(v_{E}\right)\right)$ tais que $f^{\prime}(u)$ é derivável com respeito a $v_{E}$, e para as quais existe uma função $g \in C_{0}\left(\operatorname{supp}\left(v_{E}\right)\right)$ que satisfaz a equação (2.4).

Portanto, $\mathcal{L}_{W}^{Z}: \mathcal{D}_{W}^{Z} \rightarrow C_{0}\left(\operatorname{supp}\left(v_{E}\right)\right)$ é o gerador do semigrupo $\{S(t): t \geq 0\}$ e

$$
\mathcal{L}_{W}^{Z}=\frac{d}{d v_{E}} \frac{d}{d u}
$$

Observação. Dada uma função contínua, (estritamente) crescente $W$ e uma função real $f$, definimos a derivada generalizada de $f$ em relação a $W$ por

$$
\frac{d f}{d W}(x)=\lim _{y \rightarrow x} \frac{f(y)-f(x)}{W(y)-W(x)}
$$

sempre que este limite exista.

\section{$2.2 \quad$ O Processo $Y(t, u)$}

Observação. Dos resultados obtidos em [15], temos que o processo $Y(t, u)=Y_{t}$ não é fortemente Markov com respeito à filtração $\mathcal{F}_{t}^{Y}=\sigma\left(Y_{s}: s \leq t\right)$ e, em particular, não é um processo de Feller.

Denote por $\left\{x_{j}: j \geq 1\right\}$ os pontos de salto de $W$. Temos que 


$$
W^{-1}\left(W\left(x_{j}\right)\right)=W^{-1}\left(W\left(x_{j}-\right)\right)
$$

Para achar o gerador do processo $Y_{t}$, vamos colocar mais pontos dividindo em dois pontos, os pontos de salto de $W$, e assim obteremos uma função bijetora $W_{b}$. Isto é, seja $\left\{x_{j}^{-}: j \geq 1\right\}$ e $W_{b}$ tal que

$W_{b}: \mathbb{R} \bigcup\left\{x_{j}^{-}: j \geq 1\right\} \rightarrow \operatorname{supp}\left(v_{E}\right)$ tal que $W_{b}(x)=W(x)$ para $x \in \mathbb{R}$, e $W_{b}\left(x_{j}^{-}\right)=W\left(x_{j}-\right)$ para $j \geq 1$.

A função inversa $W_{b}^{-1}: \operatorname{supp}\left(v_{E}\right) \rightarrow \mathbb{R} \bigcup\left\{x_{j}^{-}: j \geq 1\right\}$, é dada por $W_{b}^{-1}(W(x))=x, W_{b}^{-1}\left(W\left(x_{j}-\right)\right)=$ $x_{j}^{-}$.

Considere os espaços $\left(\mathbb{R} \bigcup\left\{x_{j}^{-}: j \geq 1\right\}, d_{W_{B}}\right)$ e $\left(\operatorname{supp}\left(v_{E}\right), d\right)$, onde $d_{W_{B}}$ é definida da seguinte maneira:

$$
d_{W_{B}}\left(x_{j}^{-}, x_{k}^{-}\right)=\left|W\left(x_{j}-\right)-W\left(x_{k}-\right)\right|, \quad d_{W_{B}}\left(x_{j}^{-}, y\right)=\left|W\left(x_{j}-\right)-W(y)\right| .
$$

Então $W_{b}$ é uma isometría entre os dois espaços métricos acima definidos, pois conserva as distâncias entre os pontos. Assim, os resultados obtidos no processo $Z$ valem também para o processo $U_{t}=W_{b}^{-1}\left(Z_{t}\right)$ com espaço de estados $\mathbb{R} \bigcup\left\{x_{j}^{-}: j \geq 1\right\}$.

Vamos estudar o gerador $\mathcal{L}_{W}^{U}$ do processo $U_{t}$. O domínio $\mathcal{D}_{W}^{U}$ do gerador do processo $U_{t}$ é dado por

$$
\mathcal{D}_{W}^{U}=\left\{f \circ W_{b}: f \in \mathcal{D}_{W}^{Z}\right\}
$$

onde $f \circ W_{b}$ denota a composição de $f \operatorname{com} W_{b}$.

Sejam $f, g \in C_{0}\left(\operatorname{supp}\left(v_{E}\right)\right)$ com $c=f(0)$ e $e=f^{\prime}(0)$ tais que

$$
f(u)=\int_{0}^{u} \int_{0}^{w} g(a) v_{E}(d a) d w+c+u e .
$$


Uma simples troca de variáveis permite escrever

$$
f\left(W_{b}(x)\right)=\int_{0}^{W_{b}(x)} \int_{0}^{W_{b}(y)} g\left(W_{b}(z)\right) d W^{-1}\left(W_{b}(z)\right) d W_{b}(y)+c+W_{b}(x) e .
$$

Assim, para $x \in \mathbb{R}, f \circ W_{b}$, é dada por

$$
f \circ W_{b}(x)=\int_{0}^{x} \int_{0}^{y} g \circ W_{b}(z) d(z) d W(y)+c+W(x) e
$$

e, escrevendo $F=f \circ W_{b}$ e $G=g \circ W_{b}$, segue que

$$
F(x)=\int_{0}^{x} \int_{0}^{y} G(z) d(z) d W(y)+c+e W(x) .
$$

A continuidade em $x$ permite escrever

$$
F\left(x_{j}^{-}\right)=F\left(x_{j}-\right), \quad \forall j \geq 1 .
$$

Segue que $\mathcal{D}_{W}^{U}$ é o conjunto de funções $F \in C_{0}\left(\mathbb{R} \cup\left\{x_{j}^{-}: j \geq 1\right\}\right)$ para as quais existe $G \in$ $C_{0}\left(\mathbb{R} \cup\left\{x_{j}^{-}: j \geq 1\right\}\right)$ satisfazendo a equação (2.5). Segue que

$$
\mathcal{L}_{W}^{U}=\frac{d}{d x} \frac{d}{d W} \quad \text { e } \quad \mathcal{L}_{W}^{U} F=G
$$

Retomando o processo $Y_{t}=Y(t, x)$, denotamos por $\{T(t): t \geq 0\}$ ao semigrupo do processo de Markov $Y_{t}$, cujo domínio são as funções Borel mensuráveis limitadas $f: \mathbb{R} \rightarrow \mathbb{R}$.

Seja $C_{W, 0}(\mathbb{R})$ o conjunto das funções contínuas a direita com limite a esquerda, limitadas e que se anulam em $\pm \infty$, tais que o conjunto de discontinuidades é um subconjunto do conjunto formado pelos pontos de salto de $W$. Seja $\varphi: \mathbb{R} \cup\left\{x_{j}^{-}: j \geq 1\right\} \rightarrow \mathbb{R}$ tal que $\varphi(x)=x$ para todo $x \in \mathbb{R}$ e $\varphi\left(x_{j}^{-}\right)=x_{j}$ para todo $j \geq 1$. Então podemos escrever $Y_{t}=\varphi\left(U_{t}\right)$.

Dada a função $H: \mathbb{R} \rightarrow \mathbb{R}$, defina a função

$$
H \circ \varphi: \mathbb{R} \bigcup\left\{x_{j}^{-}: j \geq 1\right\} \rightarrow \mathbb{R} .
$$


Temos que

$$
\mathcal{L}_{W}^{Y} H=\pi \mathcal{L}_{W}^{U} H \circ \varphi, \quad H \in \mathcal{D}_{W}^{Y},
$$

onde $\pi f: \mathbb{R} \rightarrow \mathbb{R}$ é definida por $\pi f(x)=f(x)$ e $f: \mathbb{R} \rightarrow \mathbb{R}$. $\mathcal{D}_{W}^{Y}$ é o conjunto de funções $H \in C_{W, 0}(\mathbb{R})$ para as quais existe $G \in C_{W, 0}(\mathbb{R})$ e constantes $c, e \in \mathbb{R}$ satisfazendo a equação

$$
H(x)=\int_{0}^{x} \int_{0}^{y} G(z) d(z) d W(y)+c+e W(x), \quad x \in \mathbb{R} .
$$

Assim, considerando o operador linear $\mathcal{L}_{W}^{Y}: \mathcal{D}_{W}^{Y} \rightarrow C_{W, 0}(\mathbb{R})$ dado por

$$
\mathcal{L}_{W}^{Y} H=\frac{d}{d x} \frac{d}{d W} H=G
$$

de (2.7) temos que $H$ satisfaz

$$
\frac{\partial H}{\partial x}\left(x_{j}+\right)=\frac{\partial H}{\partial x}\left(x_{j}-\right)
$$

$\mathrm{e}$

$$
\frac{\partial H}{\partial x}(0+)=\lambda\left[H\left(x_{j}+\right)-H\left(x_{j}-\right)\right]
$$

Mais algumas definições e resultados são necessárias para o desenvolvimento de nosso trabalho:

Definição 5 Seja $\mathcal{M}_{+}$o espaço de medidas positivas, finitas em $\mathbb{I}=[0,1)$ e $D\left([0, T], \mathcal{M}_{+}\right)$o espaço das funções contínuas à direita com limite à esquerda tomando valores em $\mathcal{M}_{+}$. Uma trajetória determinística é definida como o suporte de uma medida de probabilidade de Dirac no espaço $D\left([0, T], \mathcal{M}_{+}\right)$concentrada nesta trajetória.

Definição 6 Um conjunto é relativamente compacto se toda seqüência de pontos do conjunto possui uma subseqüência convergente (cujo limite não precisa pertencer necessariamente ao conjunto). 
Resultado 2 (Prohorov). Defina uma métrica no espaço $\mathcal{M}_{+}$introduzindo uma família densa enumerável de funções contínuas $\left\{f_{k}: k \geq 1\right\}$ em $\mathbb{I}$, e definindo a distância $\delta(.,$.$) por:$

$$
\delta(\mu, \nu)=\sum_{k=1}^{\infty} \frac{1}{2^{k}} \frac{\left|<\mu, f_{k}>-<\nu, f_{k}>\right|}{1+\left|<\mu, f_{k}>-<\nu, f_{k}>\right|},
$$

onde $<\mu, f>$ é a integral de $f$ com respeito a $\mu$.

Uma seqüência de medidas de probabilidade $\left\{Q^{N}, N \geq 1\right\}$, no espaço $D\left([0, T], \mathcal{M}_{+}\right)$, é relativamente compacta se e somente se para todo $\epsilon>0$ e todo $t \in[0, T]$, existe $K_{\epsilon, t} \subset \mathcal{M}_{+}$, conjunto compacto, tal que

$$
Q^{N}\left(K_{\epsilon, t}\right) \geq 1-\epsilon, \quad N \geq 1
$$

$e$

$$
\lim _{\gamma \rightarrow 0} \limsup _{N \rightarrow \infty} \sup _{\tau \in \mathcal{T}_{T}, \theta \leq \gamma} Q^{N}\left[\delta\left(\mu_{\tau}, \mu_{\tau+\theta}\right)>\epsilon\right]=0
$$

para todo $\epsilon>0$, onde $\mathcal{T}_{T}$ é a família de tempos de parada limitados por $T$.

Observação. Convergência fraca induz uma topologia sobre o espaço de probabilidade, e aparece na literatura com o nome de topologia fraca. Certas propriedades do espaco métrico induzido na topologia se transferem para o espaço de probabilidade, e uma aplicacão desse conceito é demonstrar que uma seqüência de probabilidades converge a uma probabilidade específica. O método geralmente empregado para demonstrar este resultado é caracterizado por 3 etapas:

- Mostrar que a seqüência é relativamente compacta.

- Mostrar que o limite é único.

- Caracterizar o limite.

Resultado 3 ([7], capítulo 2) Seja $\left\{g_{k} ; k \geq 1\right\}$ uma subfamilia densa em $C(I \Gamma)$ com $g_{1}=1$. A família de medidas de probabilidade $\left(Q^{N}\right)_{N \geq 1}$ em $D\left([0, T], \mathcal{M}_{+}\right)$é relativamente compacta se para todo inteiro positivo $k$, a família $Q^{N} g_{k}^{-1}$ é relativamente compacta. Aqui $Q^{N} g_{k}^{-1}$ é definida por

$$
Q^{N} g_{k}^{-1}[A]=Q^{N}\left[\pi^{N} ;<\pi^{N}, g_{k}>\in A\right] .
$$


Definição 7 Seja $\left\{T_{N}(t): t \geq 0\right\}$ o semigrupo associado ao processo $\left\{Y_{N}(t): t \geq 0\right\}$. Temos que para toda $F \in C_{c}(\mathbb{R})$ e $\alpha>0$, o resolvente associado ao semigrupo $\left\{T_{N}(t): t \geq 0\right\}$, que denotaremos por $R_{\alpha}^{N}$, é definido por

$$
R_{\alpha}^{N} F=\int_{0}^{\infty} e^{-\alpha t} T_{N}(t) F d t
$$

O resultado a seguir estabelece algumas propriedades e resultados de convergência do semigrupo $\left\{T_{N}(t): t \geq 0\right\}$

Resultado 4 ([15]) Fixe $F \in C_{c}(\mathbb{R})$. Então

$$
\lim _{t \rightarrow 0} \limsup _{N \rightarrow \infty} \frac{1}{N} \sum_{x \in \boldsymbol{Z}}\left|T_{N}(t) F(x / N)-F(x / N)\right|=0,
$$

e para todo $\alpha>0$,

$$
\begin{gathered}
\lim _{\alpha \rightarrow \infty} \limsup _{N \rightarrow \infty} \frac{1}{N} \sum_{x \in \boldsymbol{Z}}\left|\alpha R_{\alpha}^{N} F(x / N)-F(x / N)\right|=0, \\
\frac{1}{N} \sum_{x \in \boldsymbol{Z}}\left|\alpha R_{\alpha}^{N} F(x / N)\right| \leq \frac{1}{N} \sum_{x \in \boldsymbol{Z}}|F(x / N)| .
\end{gathered}
$$

Resultado 5 (Scheffe) Para $h \in L^{1}(\mathbb{R})$, seja $h_{n} \in L^{1}(\mathbb{R}), n \in \mathbb{N}^{+}$, uma seqüência de funções satisfazendo,

- $h_{n} \geq 0$

- $h_{n}(x) \rightarrow h(x), \quad x \in \mathbb{R}$

- $\int_{\mathbb{R}} h_{n}(x) d x \rightarrow \int_{\mathbb{R}} h(x) d x$.

Então $\lim _{n \rightarrow \infty} \int_{\mathbb{R}}\left|h_{n}(x)-h(x)\right| d x=0$

Resultado 6 ([7]) Considere uma função limitada $F \quad: \quad \mathbb{R}_{+} \times \quad E \quad \rightarrow \quad I R$, suave, isto é; com a primeira derivada contínua e a segunda derivada quadrado integrável. Para cada $x \in E, F(., x) \in C^{2}$, onde $C^{2}$ é o espaço das funções duas vezes diferenciáveis, existe uma constante finita $C>0$ tal que

$$
\sup _{(s, x)}\left|\left(\partial_{s}^{j} F\right)(s, x)\right| \leq C
$$


para $j=1,2$. Denote por $\left\{\mathcal{F}_{t}, t \geq 0\right\}$ a filtração canônica do processo de Markov $X_{t}$, isto é, $\mathcal{F}_{t}=\sigma\left(X_{s}, s \leq t\right)$, e por $L$ o gerador de tal processo. Os processos $M^{F}(t)$ e $N^{F}(t)$ definidos por

$$
\begin{array}{r}
M^{F}(t)=F\left(t, X_{t}\right)-F\left(0, X_{0}\right)-\int_{0}^{t} d s\left(\partial_{s}+L\right) F\left(s, X_{s}\right) \\
N^{F}(t)=\left(M^{F}(t)\right)^{2}-\int_{0}^{t} d s\left[L F\left(s, X_{s}\right)^{2}-2 F\left(s, X_{s}\right) L F\left(s, X_{s}\right)\right]
\end{array}
$$

são $\mathcal{F}_{t}$ martingais relativos a esta filtração.

\subsection{Estudo do Limite Hidrodinâmico}

Apresentamos a seguir a demonstração do limite hidrodinâmico do processo em estudo, sendo este o principal resultado de nosso trabalho.

Teorema 1 Seja $\rho_{0}: \mathbb{R} \rightarrow[0,1]$ um perfil de densidade inicial, contínuo, limitado e que se anula no infinito e seja $\mu^{N}$ uma seqüência de medidas produto de Bernoulli tais que:

$$
\mu^{N}\{\eta ; \eta(x)=1\}=\rho_{0}(x / N)
$$

Então, para todo $t>0$, a seqüência de medidas empíricas

$$
\pi_{t}^{N}(d u)=\frac{1}{N} \sum_{x} \eta_{t}(x) \delta_{x / N}(d u)
$$

converge em probabilidade à medida $\pi_{t}(d u)=\rho(t, u) d u$ cuja função de densidade $\rho$ é a solução da seguinte equação:

$$
\left\{\begin{array}{ll}
\partial_{t} \rho & =\Delta \rho \text { nos intervalos }\left(\gamma_{j}, \gamma_{j+1}\right) \\
\partial_{x} \rho\left(t, x_{j}+\right) & =\partial_{x} \rho\left(t, x_{j}-\right) \\
\partial_{x} \rho\left(t, x_{j}+\right) & =\lambda\left[\rho\left(t, x_{j}-\right)-\rho\left(t, x_{j}+\right)\right]
\end{array},\right.
$$

onde $\rho(t, u)=T(t) \rho_{0}(u)$ e $\Delta \rho$ é o Laplaciano de $\rho$. 


\section{Estratégia da Demonstração}

Vamos demonstrar o teorema em duas etapas. Primeiro demonstraremos que a medida empírica dada no Teorema 1 converge em probabilidade à medida $\pi_{t}(d u)=\rho(t, u) d u$ cuja função de densidade é a solução da equação (2.11). Depois demonstraremos que existe uma única solução fraca de (2.11).

\section{Etapa 1:}

Para uma medida positiva $\pi$ de massa total finita e para funções $G \in C_{c}(\mathbb{R})$, denotamos por $\langle\pi, G>$ à integral de $G$ com respeito a $\pi$, isto é,

$$
<\pi, G>=\int G(u) \pi(d u) .
$$

Vamos provar que a medida empírica resolve a equação (2.11) em sentido fraco. Isto é, vamos provar que

$$
\pi_{t}^{N} \rightarrow \pi_{t} \quad \text { quando } \quad N \rightarrow \infty
$$

em probabilidade, onde

$$
\begin{aligned}
\pi_{t}^{N}(d u) & =\pi^{N}\left(\eta_{t}, d u\right) \\
& =\frac{1}{N} \sum_{x \in \boldsymbol{Z}} \eta_{t}(x) \delta_{x / N}(d u)
\end{aligned}
$$

Fixe uma realização de $W$ e $T>0$. Para cada medida de probabilidade $\mu \in\{0,1\}^{\boldsymbol{Z}}$, denote por $\mathbb{Q}_{\mu}^{W}$ a medida no espaço $D\left([0, T], \mathcal{M}_{+}\right)$(espaço das funções contínuas a direita com limites à esquerda tomando valores em $\mathcal{M}_{+}$), induzida pela medida $\mu$. Considere o processo de Markov , evoluindo segundo o gerador

$$
\begin{aligned}
\left(\mathcal{L}_{\mathcal{N}} f\right)(\eta)(x)=c_{x} \eta(x)(1-\eta(x+1))\left\{f\left(\sigma^{x, x+1} \eta\right)-f(\eta)\right\}+ \\
c_{x-1} \eta(x)(1-\eta(x-1))\left\{f\left(\sigma^{x, x-1} \eta\right)-f(\eta)\right\},
\end{aligned}
$$

acelerado por $N^{2}$ e começando em $\mu^{N}$. Aqui $\sigma^{x, x+1} \eta$ é a configuração obtida de $\eta$ ao intercambiar as variáveis $\eta(x)$ e $\eta(x+1)$, isto é,

$$
\sigma^{x, x+1} \eta(y)= \begin{cases}\eta(x+1) & \text { se } y=x, \\ \eta(x) & \text { se } y=x+1, \\ \eta(y) & \text { caso contrário }\end{cases}
$$


Seja $\mathbb{Q}^{W}$ a medida de probabilidade em $D\left([0, T], \mathcal{M}_{+}\right)$concentrada no caminho determinístico $\pi_{t}(d u)=\rho(t, u) d u$, onde $\rho(t, u)=T(t) \rho_{0}(u)$. Vamos provar que para cada $t$ fixado, $\pi_{t}^{N}$ converge em probabilidade para $\rho(t, u) d u$, onde $\rho(t, u)=T(t) \rho_{0}(u)$ é a solução da equação $(2.11)$ com condição inicial $\rho_{0}$. Isto é, se $\mathbb{P}_{\mu^{N}}^{W, N}$ é a distribuição no espaço $D\left([0, T],\{0,1\}^{\boldsymbol{Z}}\right)$ do processo de exclusão $\left\{\eta_{t}: t \geq 0\right\}$ com distribuição inicial $\mu^{N}$ e gerador $\mathcal{L}_{\mathcal{N}}$ acelerado por $N^{2}$, vamos mostrar que

$$
\lim _{N \rightarrow \infty} \mathbb{P}_{\mu^{N}}^{W, N}\left[\sup _{0 \leq t \leq T}\left|\frac{1}{N} \sum_{x \in \boldsymbol{Z}} G(x / N) \eta_{t N^{2}}(x)-\int_{\mathbb{R}} G(u) \rho(t, u) d u\right|>\epsilon\right]
$$

é igual a zero para toda função $G \in C_{c}(\mathbb{R})$ e todo $\epsilon>0$.

Para levar a termo nosso objetivo, vamos provar que $\pi_{t}^{N}$ converge em distribuição à medida de probabilidade $\mathbb{Q}^{W}$ concentrada no caminho determinístico $\{\rho(t, u) d u, 0 \leq t \leq T\}$, e com isso argumentar, que convergência em distribuição, a uma trajetória determinística contínua implica convergência em probabilidade para $0 \leq t \leq T$.

Provar o comportamento hidrodinâmico do processo então é mostrar que $\mathbb{Q}_{\mu}^{W}$ converge para a medida de Dirac concentrada na solução da equação (2.11). Para isso, vamos seguir os seguintes passos:

1. Mostrar que $Q_{\mu}^{W}$ é relativamente compacta (usando o criterio de Prohorov) e, depois mostrar que todas as subseqüências convergentes convergem ao mesmo limite.

2. Examinar os subconjuntos compactos de $D\left([0, T], \mathcal{M}_{+}\right)$onde precisamos que seja factível considerar medidas de Dirac concentradas numa trajetória. Para caracterizar todos os pontos limite de $\mathbb{Q}_{\mu}^{W}$ usaremos o fato de que, sob $\mathbb{Q}_{\mu^{N}}^{W}$, é satisfeita a seguinte identidade:

$$
\left\langle\pi_{t}^{N}, f_{\alpha, N}\right\rangle=\left\langle\pi_{0}^{N}, f_{\alpha, N}\right\rangle+\int_{0}^{t} N^{2} \mathcal{L}_{\mathcal{N}}\left\langle\pi_{s}^{N}, f_{\alpha, N}\right\rangle d s+M_{t}^{\alpha, N}
$$

onde $M_{t}^{\alpha, N}$ é martingal com respeito à filtração $\mathcal{F}_{t}=\sigma\left(\eta_{s}, s \leq t\right)$ e $f_{\alpha, N}=R_{\alpha}^{N} F$.

Etapa 2: Para concluir a prova, devemos mostrar que $M_{t}^{\alpha, N} \rightarrow 0$ quando $N \uparrow \infty$, e que existe uma solução única para a equação (2.11). A prova do limite hidrodinâmico termina com a prova de unicidade para as soluções fracas da equação diferencial parcial que descreve a evolução macroscópica do sistema. 
Segue que $Q_{\mu}^{W}$ tem um único limite $\mathbb{Q}^{W}$, que é a medida de probabilidade concentrada na única solução de (2.11).

\section{Demonstração do Teorema}

Seja $T>0$, fixe uma realização de $W$ e considere uma seqüência de medidas de probabilidade $\mathbb{Q}_{\mu^{N}}^{W}$ em $D\left([0, T], \mathcal{M}_{+}\right)$, correspondentes ao processo de Markov $\pi_{t}^{N}$ definido na equação (2.12), acelerado por $N^{2}$ e começando em $\mu^{N}$. Assim como consideramos duas escalas de espaço: $\mathbb{Z}, \mathbb{Z}_{N}$, consideramos um tempo macroscopico $t$ e um tempo microscopico acelerado por $N^{2}$ com respeito a $t$, isto é $t N^{2}$.

- Primeiro vamos demonstrar que $\mathbb{Q}_{\mu^{N}}^{W}$ é relativamente compacta. Do Resultado 3, é suficiente estudar a compacidade relativa para $\left\langle\pi_{t}^{N}, g_{k}>\right.$, onde $\left\{g_{k}, k \geq 1\right\}$ é uma subfamília densa enumerável de $C(\mathbb{R})$. Assim, introduzimos o operador linear limitado em $C_{c}(\mathbb{R}), R_{\alpha}^{N}=$ $\left(\alpha-\mathcal{L}_{W}^{Y_{N}}\right)^{-1}$, o resolvente de $\mathcal{L}_{W}^{Y_{N}}$, onde $\mathcal{L}_{W}^{Y_{N}}$ é o gerador associado ao processo $\left\{Y_{N}(t): t \geq 0\right\}$.

Para toda $F \in C_{c}(\mathbb{R})$ e $\alpha>0$, temos

$$
R_{\alpha}^{N} F=\int_{0}^{\infty} e^{-\alpha t} T_{N}(t) F d t .
$$

Pelo Resultado 3 basta mostrar que a seqüência de medidas correspondentes ao processo $\left\langle\pi_{t}^{N}, R_{\alpha}^{N} F\right\rangle$ é relativamente compacta em $D\left([0, T], \mathcal{M}_{+}\right)$para toda $F \in C_{c}(\mathbb{R})$.

Fixe $F \in C_{c}(\mathbb{R})$ e considere $f_{\alpha, N}=R_{\alpha}^{N} F$. Denote por $\mathbb{Q}_{\mu^{N}}^{W} f_{\alpha, N}^{-1}$ a medida de probabilidade em $D([0, T], \mathbb{R})$.

Temos que $N^{2} \mathcal{L}_{\mathcal{N}}\left\langle\pi_{t}^{N}, F\right\rangle=\left\langle\pi_{t}^{N}, \mathbb{L}_{N} F\right\rangle$, onde $\mathcal{L}_{\mathcal{N}}$ é o operador definido na equação (2.13) e $\mathbb{L}_{N}$ é o operador definido na equação (2.1). Seja $f\left(\eta_{t}\right)=\frac{1}{N} \sum_{x \in \mathbb{Z}} F\left(\frac{x}{N}\right) \eta_{t}(x)$. Segue que

$$
\begin{aligned}
f\left(\sigma^{x, x+1} \eta_{t}\right)-f\left(\eta_{t}\right)= & \frac{1}{N}\left[F\left(\frac{x+1}{N}\right)-F\left(\frac{x}{N}\right)\right] \eta_{t}(x)+ \\
& \frac{1}{N}\left[F\left(\frac{x}{N}\right)-F\left(\frac{x+1}{N}\right)\right] \eta_{t}(x+1) .
\end{aligned}
$$


Portanto,

$$
\begin{aligned}
\mathcal{L}_{\mathcal{N}}\left\langle\pi_{t}^{N}, F\right\rangle= & \frac{1}{N} \sum_{x \in \mathbb{Z}} c_{x}\left\{F\left(\frac{x+1}{N}\right)-F\left(\frac{x}{N}\right)\right\} \eta_{t}(x)+ \\
& \frac{1}{N} \sum_{x \in \mathbb{Z}} c_{x-1}\left\{F\left(\frac{x-1}{N}\right)-F\left(\frac{x}{N}\right)\right\} \eta_{t}(x) \\
= & N^{-2}\left\langle\pi_{t}^{N}, \mathbb{L}_{N} F\right\rangle
\end{aligned}
$$

Temos que, sob $Q_{\mu^{N}}^{W}$, a seguinte identidade é satisfeita

$$
\left\langle\pi_{t}^{N}, f_{\alpha, N}\right\rangle=\left\langle\pi_{0}^{N}, f_{\alpha, N}\right\rangle+\int_{0}^{t} N^{2} \mathcal{L}_{\mathcal{N}}\left\langle\pi_{s}^{N}, f_{\alpha, N}\right\rangle d s+M_{t}^{\alpha, N},
$$

onde $M_{t}^{\alpha, N}$ é um martingal com respeito à filtração $\mathcal{F}_{t}=\sigma\left(\eta_{s}, s \leq t\right)$. A equação (2.14) permite escrever

$$
\begin{aligned}
\left\langle\pi_{t}^{N}, f_{\alpha, N}\right\rangle & =\left\langle\pi_{0}^{N}, f_{\alpha, N}\right\rangle+\int_{0}^{t}\left\langle\pi_{s}^{N}, I_{N} f_{\alpha, N}\right\rangle d s+M_{t}^{\alpha, N} \\
& =\left\langle\pi_{0}^{N}, f_{\alpha, N}\right\rangle+\int_{0}^{t}\left\{\alpha\left\langle\pi_{s}^{N}, f_{\alpha, N}\right\rangle-\left\langle\pi_{s}^{N}, F\right\rangle\right\} d s+M_{t}^{\alpha, N}
\end{aligned}
$$

Para verificar o limite que corresponde à equação (2.10) vamos estudar o que acontece com o segundo e o terceiro termos da direita da equação (2.16).

Defina $N_{t}^{\alpha, N}=\left(M_{t}^{\alpha, N}\right)^{2}-\int_{0}^{t} A_{s}^{\alpha, N} d s$ onde

$$
A_{t}^{\alpha, N}=N^{2} \mathcal{L}_{\mathcal{N}}\left\langle\pi_{t}^{N}, f_{\alpha, N}\right\rangle^{2}-2 N^{2}\left\langle\pi_{t}^{N}, f_{\alpha, N}\right\rangle \mathcal{L}_{\mathcal{N}}\left\langle\pi_{t}^{N}, f_{\alpha, N}\right\rangle
$$

Da equação (2.16) e pelo Resultado 6 , segue que $N_{t}^{\alpha, N}$ é um martingal.

Agora, $A_{s}^{\alpha, N}$ pode ser escrito como

$$
-\left\langle\pi_{s}^{N}, f_{\alpha, N}\right\rangle\left\langle\pi_{s}^{N}, \Pi_{N} f_{\alpha, N}\right\rangle
$$


Segue que

$$
\begin{aligned}
A_{s}^{\alpha, N}= & {\left[\sum_{x \in \mathbb{Z}} c_{x}\left\{f_{\alpha, N}(x+1 / N)-f_{\alpha, N}(x / N)\right\}\left\{\eta_{s}(x)-\eta_{s}(x+1)\right\}\right] } \\
& \times\left[\sum_{x \in \mathbb{Z}} f_{\alpha, N}(x / N) \eta_{s}(x)\right] \\
= & \frac{1}{N^{2}} \sum_{x \in \mathbb{Z}} c_{x}\left(\nabla_{N} f_{\alpha, N}\right)(x / N)^{2}\left\{\eta_{s}(x+1)-\eta_{s}(x)\right\}^{2}
\end{aligned}
$$

onde $\nabla_{N}$ é o Gradiente discreto definido por:

$$
\left(\nabla_{N} g\right)(x / N)=N\{g(x+1 / N)-g(x / N)\}
$$

Para $\tau \in \mathcal{T}_{T}$ e $\theta \leq \gamma$, como na equação (2.10), temos

$$
\mathbb{E}_{\mathscr{Q}_{\mu_{N}}^{W, N}}\left[\left(M_{\tau+\theta}^{\alpha, N}-M_{\tau}^{\alpha, N}\right)^{2}\right]=\mathbb{E}_{\mathscr{Q}_{\mu_{N}}^{W, N}}\left[\int_{\tau}^{\tau+\theta} A_{s}^{\alpha, N} d s\right]
$$

Como $f_{\alpha, N}$ é a solução de

$$
\alpha f_{\alpha, N}-\mathbb{L}_{N}^{X^{\prime}} f_{\alpha, N}=F
$$

onde $I_{N}^{X^{\prime}}$ é o gerador do processo $N^{-1} X_{N}(t)$, se multiplicarmos a identidade acima por $N^{-1} f_{\alpha, N}$ e somarmos em $x$ obtemos

$$
\begin{aligned}
\frac{\alpha}{N} \sum_{x \in \mathbb{Z}} f_{\alpha, N}(x / N)^{2} & -\frac{1}{N} \sum_{x \in \boldsymbol{Z}} f_{\alpha, N}(x / N) \mathbb{L}_{N}^{N^{-1} X} f_{\alpha, N}(x / N) \\
& =\frac{1}{N} \sum_{x \in \mathbb{Z}} f_{\alpha, N}(x / N) F(x / N) .
\end{aligned}
$$

Além disso

$$
\frac{1}{N} \sum_{x \in \boldsymbol{Z}} f_{\alpha, N}(x / N) \mathbb{L}_{N}^{X^{\prime}} f_{\alpha, N(x / N)}
$$


é igual a

$$
\begin{aligned}
N \sum_{x \in \mathbb{Z}} c_{x} f_{\alpha, N}(x / N) \times & \left\{f_{\alpha, N}\left(\frac{x+1}{N}\right)-f_{\alpha, N}(x / N)\right\}+ \\
& c_{x-1} f_{\alpha, N}(x / N)\left\{f_{\alpha, N}\left(\frac{x-1}{N}\right)-f_{\alpha, N}(x / N)\right\} \\
= & -\frac{1}{N} \sum_{x \in \mathbb{Z}} c_{x}\left(\nabla_{N} f_{\alpha, N}\right)(x / N)^{2}
\end{aligned}
$$

onde $\mathbb{L}_{N}^{X^{\prime}}$ é o gerador do processo $N^{-1} X_{N}(t)$.

Substituindo (2.20) em (2.19), segue que

$$
\begin{gathered}
\frac{\alpha}{N} \sum_{x \in \mathbb{Z}} f_{\alpha, N}(x / N)^{2}+\frac{1}{N} \sum_{x \in \boldsymbol{Z}} c_{x}\left(\nabla_{N} f_{\alpha, N}\right)(x / N)^{2}= \\
\frac{1}{N} \sum_{x \in \mathbb{Z}} f_{\alpha, N}(x / N) F(x / N) .
\end{gathered}
$$

Do Resultado 4 e aplicando a desigualdade de Cauchy-Schwarz, temos

$$
\frac{\alpha}{N} \sum_{x \in \mathbb{Z}} f_{\alpha, N}(x / N)^{2}+\frac{1}{N} \sum_{x \in \boldsymbol{Z}} c_{x}\left(\nabla_{N} f_{\alpha, N}\right)(x / N)^{2} \leq \frac{1}{\alpha N} \sum_{x \in \boldsymbol{Z}} F(x / N)^{2} .
$$

Agora, como $F \in C_{c}(\mathbb{R})$, temos que $\frac{1}{N} \sum_{x \in \mathbb{Z}} F(x / N)^{2}$ é limitada uniformemente em $N$. Logo,

$$
\sup _{N} \frac{1}{N} \sum_{x \in \mathbb{Z}} c_{x}\left(\nabla_{N} f_{\alpha, N}\right)(x / N)^{2} \leq \frac{C(f)}{\alpha}
$$

onde $C(f)>0$ é uma constante finita que depende só da função $f$.

Substituindo (2.17) em (2.18), e fazendo uso da desigualdade (2.21), segue que (2.18) é limitado superiormente por

$$
\mathbb{E}_{Q_{\mu_{N}}^{W, N}}\left[\int_{\tau}^{\tau+\theta} A_{s}^{\alpha, N} d s\right] \leq \frac{C(f) \theta}{\alpha N}
$$

Por outro lado, retomando o segundo termo da direita da equação (2.16), o Resultado 4, nos permite afirmar que 


$$
\left|\int_{\tau}^{\tau+\theta}\left\{\alpha\left\langle\pi_{s}^{N}, f_{\alpha, N}\right\rangle-\left\langle\pi_{s}^{N}, F\right\rangle\right\} d s\right| \leq C(f) \theta
$$

Assim, como $\tau \in \mathcal{T}_{T}$, o segundo termo da direita da equação satisfaz a segunda parte do resultado de Prohorov.

Com isto, e usando a desigualdade de Doob e as desigualdades (2.22) e (2.23), provamos (2.10). Temos que

$$
\lim _{\gamma \rightarrow 0} \limsup _{N \rightarrow \infty} \sup _{\tau \in \mathcal{T}_{T}, \theta \leq \gamma} \mathbb{Q}_{\mu^{N}}^{W}\left[\left|\left\langle\pi_{t}^{N}, R_{\alpha}^{N} F\right\rangle,\left\langle\pi_{t+\theta}^{N}, R_{\alpha}^{N} F\right\rangle\right|>\epsilon\right]=0
$$

Além disso, como $\sup _{N} \frac{\alpha}{N} \sum_{x \in \mathbb{Z}}\left|f_{\alpha, N}(x / N)\right| \leq C(F)$, segue que

$$
\sup _{t \geq 0} \sup _{N \geq 1}\left|\left\langle\pi_{t}^{N}, R_{\alpha}^{N} F\right\rangle\right| \leq \frac{C(F)}{\alpha}
$$

Portanto, da desigualdade acima e da Equação (2.24), o Teorema de Prohorov permite afirmar que $\left\langle\pi_{t}^{N}, R_{\alpha}^{N} F\right\rangle$ é relativamente compacta.

Do Resultado 3, segue que $Q_{\mu_{N}}^{W}$ é relativamente compacta, pois para toda $f \in C_{c}(\mathbb{R})$, considerando que existe no máximo uma partícula por sítio, segue que,

$$
\left|\left\langle\pi_{t}^{N}, R_{\alpha}^{N}(\alpha f)\right\rangle-\left\langle\pi_{t}^{N}, f\right\rangle\right| \leq \frac{1}{N} \sum_{x \in \mathbb{Z}}\left|\alpha f_{\alpha, N}(x / N)-f(x / N)\right|
$$

Do Resultado 4,

$$
\lim _{N \rightarrow \infty} \mathbb{P}_{\mu_{N}}^{W, N}\left[\sup _{0 \leq t \leq T}\left|\left\langle\pi_{t}^{N}, R_{\alpha}^{N}(\alpha f)\right\rangle-\left\langle\pi_{t}^{N}, f\right\rangle\right|>\epsilon\right]=0 .
$$

- Vamos agora caracterizar os pontos limites de $\mathbb{Q}_{\mu_{N}}^{W}$. O objetivo é demonstrar que dada $F \in$ $C_{c}(\mathbb{R})$

$$
\lim _{N \rightarrow \infty} \mathbb{P}_{\mu_{N}}^{W, N}\left[\left|\left\langle\pi_{t}^{N}, F\right\rangle-\int_{\mathbb{R}} F(x) \rho(t, x) d x\right|>\epsilon\right]=0,
$$

para todo $0 \leq t \leq T, \epsilon>0$, onde $\rho(t, x)=T(t) \rho_{0}(x)$ satisfaz a equação (2.11). 
Lema 1 Fixe uma realização do processo $W$. Para $F \in C_{c}(\mathbb{R})$ e $t \geq 0$,

$$
\lim _{N \rightarrow \infty} \mathbb{E}_{\mu_{N}}^{W, N}\left[\frac{1}{N} \sum_{x \in \boldsymbol{Z}} F(x / N) \eta_{t}(x)-\frac{1}{N} \sum_{x \in \boldsymbol{Z}} T_{N}(t) F(x / N) \eta_{0}(x)\right]^{2}=0 .
$$

\section{Demonstração}

Considere um conjunto de processos de Poisson independentes $\left(N_{x}(t): x \in \mathbb{Z}\right)$ com parâmetro $c_{x}$.

Os resultados de K. Nagy em [18] (2002) afirmam que o processo $\eta_{t}$ pode ser escrito como

$$
d \eta_{t}(x)=\left(\eta_{t^{-}}(x+1)-\eta_{t^{-}}(x)\right) d N_{x}(t)+\left(\eta_{t^{-}}(x-1)-\eta_{t^{-}}(x)\right) d N_{x-1}(t) .
$$

Seja $M_{t}^{x}=M_{t}^{x-1, x}-M_{t}^{x, x+1}, \operatorname{com} M_{0}^{x, x+1}=0 \mathrm{e}$

$$
d M_{t}^{x-1, x}=\left(\eta_{t-}(x)-\eta_{t-}(x-1)\right) d\left(N_{x}(t)-c_{x} t\right) .
$$

A equação (2.26) pode ser escrita como

$$
\eta_{t}(x)=\sum_{y \in \mathbb{Z}} p_{t}^{N}(x, y) \eta_{0}(y)+\sum_{y \in \mathbb{Z}} \int_{0}^{t} p_{t-s}^{N}(x, y) d M_{s}^{y},
$$

onde para $x, y \in \mathbb{Z}, p_{t}^{N}(x, y)=P\left[X_{N}(t, x)=y\right]$ são as probabilidades de transição do passeio aleatório $X_{N}$. Como $p_{t}^{N}$ é simétrica,

$$
\begin{aligned}
\frac{1}{N} \sum_{x \in \mathbb{Z}} F(x / N) \eta_{t}(x)= & \frac{1}{N} \sum_{x \in \boldsymbol{Z}}\left(T_{N}(t) F\right)(x / N) \eta_{0}(x)+ \\
& \frac{1}{N} \sum_{y \in \mathbb{Z}} \int_{0}^{t}\left(T_{N}(t-s) F\right)(y / N) d M_{s}^{y}
\end{aligned}
$$

Para demonstrar o lema basta demonstrar que

$$
\lim _{N \rightarrow \infty} \mathbb{E}_{\mu^{N}}^{W, N}\left[\frac{1}{N} \sum_{y \in \mathbb{Z}} \int_{0}^{t}\left(T_{N}(t-s) F\right)(y / N) d M_{s}^{y}\right]^{2}=0 .
$$


Temos que

$$
\begin{aligned}
\frac{1}{N} \sum_{y \in \mathbb{Z}} \int_{0}^{t}\left(T_{N}(t-s) F\right)(y / N) d\left(M_{s}^{y-1, y}-M_{s}^{y, y+1}\right)= \\
\left.\frac{1}{N} \sum_{y \in \mathbb{Z}} \int_{0}^{t}\left[\left(T_{N}(t-s) F\right)(y / N)-T_{N}(t-s) F\right)(y+1 / N)\right] d M_{s}^{y-1, y} .
\end{aligned}
$$

Além disso, $N_{x}(t)$ é um processo de Poisson com parâmetro $c_{x} ; \operatorname{logo}, N_{x}(t)-c_{x} t$ é um martingal para todo $x \in \mathbb{Z}$. Como $\left(\eta_{t^{-}}(x+1)-\eta_{t^{-}}(x)\right)$ é previsível com respeito a $\mathcal{F}_{t}=\sigma\left\{N_{x}(s)-c_{x} s, s \leq\right.$ $t\}, M_{s}^{x-1, x}$ também é um martingal e portanto $M_{s}^{x}$ também. Observe que $N_{x}(t)-c_{x} t, x \in \mathbb{Z}$ são $\mathcal{F}_{t}$ martingais independentes, com incrementos independentes, e com variação quadrática igual a $c_{x} t$. Segue que

$$
\begin{gathered}
\mathbb{E}_{\mu^{N}}^{W, N}\left\{\frac{1}{N} \sum_{y \in \mathbb{Z}} \int_{0}^{t}\left[T_{N}(t-s) F(y / N)-T_{N}(t-s) F(y+1 / N)\right]\right. \\
\left.\times(\eta(y+1)-\eta(y))_{s^{-}} d\left(N_{y}(s)-c_{y} s\right)\right\}^{2} \\
=\frac{N^{2}}{N^{2}} \sum_{y \in \mathbb{Z}} c_{y} \mathbb{E}_{\mu^{N}}^{W, N} \int_{0}^{t}\left[\left(T_{N}(t-s) F\right)(y / N)-\left(T_{N}(t-s) F\right)(y+1 / N)\right]^{2} \\
\times\left(\eta_{s^{-}}(y+1)-\eta_{s^{-}}(y)\right)^{2} d s \\
\left.\leq \sum_{y \in \mathbb{Z}} c_{y} \int_{0}^{t}\left[\left(T_{N}(t-s) F\right)(y / N)-T_{N}(t-s) F\right)(y+1 / N)\right]^{2} d s .
\end{gathered}
$$

A equação de Kolmogorov para $T_{N}(t) F$ permite escrever

$$
\begin{aligned}
\partial_{t} T_{N}(t) F(x / N)= & N^{2} c_{x}\left(T_{N}(t) F(x+1 / N)-T_{N}(t) F(x / N)\right) \\
& +N^{2} c_{x-1}\left(T_{N}(t) F(x-1 / N)-T_{N}(t) F(x / N)\right) .
\end{aligned}
$$

Portanto, 


$$
\begin{aligned}
& \partial_{t} \sum_{x \in Z}\left(T_{N}(t) F(x / N)\right)^{2}=2 \sum_{y \in Z} T_{N}(t) F(x / N) \partial_{t} T_{N}(t) F(x / N) \\
= & 2 N^{2} \sum_{x \in Z} c_{x}\left[T_{N}(t) F(x / N)\left(T_{N}(t) F(x+1 / N)-T_{N}(t) F(x / N)\right)\right. \\
& \left.+\left(T_{N}(t) F(x / N)-T_{N}(t) F(x+1 / N)\right)\right] \\
= & -2 N^{2} \sum_{x \in Z} c_{x}\left(T_{N}(t) F(x / N)-T_{N}(t) F(x+1 / N)\right)^{2}
\end{aligned}
$$

e segue que

$$
\begin{aligned}
\int_{0}^{t} \sum_{y \in \mathbb{Z}} c_{y}\left[\left(T_{N}(t-s) F\right)(y / N)-\left(T_{N}(t-s) F\right)(y+1 / N)\right]^{2} d s \\
=\frac{1}{N^{2}} \sum_{y \in \mathbb{Z}}\left[\left(T_{N}(0) F(x / N)\right)^{2}-\left(T_{N}(t) F(x / N)\right)^{2}\right] \\
\leq \frac{1}{N^{2}} \sum_{y \in \mathbb{Z}}\left(T_{N}(0) F(x / N)\right)^{2} \\
=\frac{1}{N^{2}} \sum_{y \in \mathbb{Z}} F^{2}(x / N) .
\end{aligned}
$$

Como $F \in C_{c}(\mathbb{R}), \lim _{N \rightarrow \infty} \frac{1}{N^{2}} \sum_{y \in \boldsymbol{Z}} F^{2}(x / N)=0$. A equação (2.30) e a equação (2.29) são suficientes para provar o lema.

Voltemos agora à equação (2.25)

$$
\lim _{N \rightarrow \infty} \mathbb{P}_{\mu_{N}}^{W, N}\left[\left|\left\langle\pi_{t}^{N}, F\right\rangle-\int_{\mathbb{R}} F(u) \rho(t, u) d u\right|>\epsilon\right]=0
$$

Nosso objetivo é demonstrar que para $F \in C_{c}(\mathbb{R})$ e para todo $0 \leq t \leq T, \epsilon>0$, o limite acima é satisfeito. Isto é, do Lema 1, precisamos mostrar que

$$
\lim _{N \rightarrow \infty} \mu_{N}\left[\left|\frac{1}{N} \sum_{x \in \mathbb{Z}} T_{N}(t) F(x / N) \eta(x)-\int_{\mathbb{R}} F(u) \rho(t, u) d u\right|>\epsilon\right]=0 .
$$


Sem perda de generalidade, assuma que $F \geq 0$. Note que

$$
\int F(u) \rho(t, u) d u=\int T(t) F(u) \rho_{0}(u) d u .
$$

Como a configuração inicial do processo de exclusão satisfaz

$$
\lim _{N \rightarrow \infty} \frac{1}{N} \sum_{x \in \mathbb{Z}} F(x / N) \eta_{0}(x)=\int F(u) \rho_{0}(u) d u
$$

em probabilidade, para toda $F \in C_{c}(\mathbb{R})$, e um perfil inicial $\rho_{0}$ dado, é suficiente mostrar que

$$
\lim _{N \rightarrow \infty} \mathbb{E}_{\mu^{N}} \frac{1}{N} \sum_{x \in \mathbb{Z}}\left|T_{N}(t) F(x / N)-T(t) F(x / N)\right| \eta_{0}(x)=0 .
$$

Como temos no máximo uma partícula por sítio,

$\mathbb{E}_{\mu^{N}} \frac{1}{N} \sum_{x \in \mathbb{Z}}\left|T_{N}(t) F(x / N)-T(t) F(x / N)\right| \eta_{0}(x)$

$\leq \frac{1}{N} \sum_{x \in \mathbb{Z}}\left|T_{N}(t) F(x / N)-T(t) F(x / N)\right|$.

Temos que $T_{N}(t) F$ e $T(t) F$ satisfazem as hipoteses do Resultado 5, pois $T_{N}(t) F \geq 0$ e $T_{N}(t) F(x)$ converge ponto a ponto para $T(t) F(x)$, para todo $x \in \mathbb{R}$, onde $T_{N}(t) F(x):=$ $T_{N}(t) F(\lceil x N\rceil / N)$ (do Resultado 2.6 temos que $\mathrm{T}(\mathrm{t})$ é um semigrupo de contração fortemente contínuo em $C_{W, 0}(\mathbb{R})$ ). Como $T(t) F(x)=\int p_{t}(x, y) F(y) d y$, onde $p_{t}(x, y)$ é a função de transição do processo $Y_{t}$, então $\int_{\mathbb{R}} T(t) F(u) d u=\int_{\mathbb{R}} F(u) d u$, e portanto é satisfeito o terceiro item do Resultado 5, pois

$$
\begin{aligned}
\int_{\mathbb{R}} T_{N}(t) F(u) & =\frac{1}{N} \sum_{x \in \boldsymbol{Z}} \sum_{y \in \mathbb{Z}} F(x / N) p_{t}^{N}(x, y) F(x / N) \\
& =\frac{1}{N} \sum_{y \in \mathbb{Z}} F(y / N) \sum_{x \in \mathbb{Z}} p_{t}^{N}(y, x)=\frac{1}{N} \sum_{y \in \mathbb{Z}} F(y / N) .
\end{aligned}
$$

$T_{N}(t) F$ e $T(t) F$ satisfazem as hipoteses do Resultado 5 e, portanto

$$
\lim _{N \rightarrow \infty} \frac{1}{N} \sum_{x \in \mathbb{Z}}\left|T_{N}(t) F(x / N)-T(t) F(x / N)\right|=0 .
$$

Provamos, portanto (2.33), (2.32) e (2.25). 
Como provamos que $Q_{\mu^{N}}^{W}$ é relativamente compacta e além disso caracterizamos os pontos limite desta seqüência, segue que $Q_{\mu^{N}}^{W}$ converge para $\mathbb{Q}^{W}$ quando $N \uparrow \infty$.

A demonstração do Teorema 1 segue da convergêngia de $Q_{\mu^{N}}^{W}$ para $Q^{W}$ quando $N \uparrow \infty$ pois, como $\int_{\mathbb{R}} F(u) \rho(t, u) d u$ é uma função contínua em $t \geq 0$, e se $G:[0, T] \rightarrow \mathbb{R}$ é também uma função contínua, a aplicação de $D\left([0, T], \mathcal{M}_{+}\right) \rightarrow \mathbb{R}$ que associa à trajetória $\left\{\pi_{t}, 0 \leq t \leq T\right\}$ o número $\sup _{0 \leq t \leq T}\left|<\pi_{t}, F>-G(t)\right|$, é portanto contínua na topologia de Skorohod. Segue que

$$
\mathbb{Q}^{W}\left\{\pi: \pi_{t}(d u)=\pi_{t}(u) d u\right\}=1
$$

A demonstração do comportamento hidrodinâmico precisa da demonstração de unicidade para as soluções da equação diferencial parcial que descreve a evolução macroscópica do sistema.

Para tanto, considere o espaço das funções quadrado integráveis que notaremos por $L^{2}$. Considere um perfil inicial $\rho_{0} \in L^{2}$ e seja $R_{\alpha}=\left(\alpha-\mathcal{L}_{W}^{Y}\right)^{-1}$, o resolvente de $\mathcal{L}_{W}^{Y}$, onde $\mathcal{L}_{W}^{Y}$ é o gerador associado ao processo $\{Y(t): t \geq 0\}$.

Sejam $\rho^{1}$ e $\rho^{2}$ duas soluções de (2.11). Seja $\overline{\rho_{t}}=\rho_{t}^{1}-\rho_{t}^{2}$. Segue que

$$
\begin{aligned}
\partial_{t}\left\langle\bar{\rho}_{t}, R_{\alpha} \bar{\rho}_{t}\right\rangle & =2\left\langle\bar{\rho}_{t}, \mathcal{L}_{W}^{Y} R_{\alpha} \bar{\rho}_{t}\right\rangle \\
& =\left\langle\bar{\rho}_{t}{ }^{2}\right\rangle+\alpha\left\langle\bar{\rho}_{t}, R_{\alpha} \bar{\rho}_{t}\right\rangle
\end{aligned}
$$

Isto é,

$$
\partial_{t}\left\langle\bar{\rho}_{t}, R_{\alpha} \bar{\rho}_{t}\right\rangle \leq \alpha\left\langle\bar{\rho}_{t}, R_{\alpha} \bar{\rho}_{t}\right\rangle
$$

Portanto,

$$
\left\langle\bar{\rho}_{t}, \bar{\rho}_{t}\right\rangle \leq\left\langle\overline{\rho_{0}}, \overline{\rho_{0}}\right\rangle e^{\alpha t}
$$

Assim, concluímos que $\rho^{1}=\rho^{2}$, o que define a unicidade.

Do Teorema 1 podemos obter o limite hidrodinámico "annealed"para o processo de exclusão em $\{0,1\}^{\mathbb{Z}}$ tal que, com taxa $\beta_{x}$, os valores das variáveis $\eta(x)$ e $\eta(x+1)$ são intercambiados.

Dado $T>0$ e uma medida de probabilidade $\mu$ em $\{0,1\}^{\boldsymbol{Z}}$, seja $\mathbb{P}_{\mu}^{\beta, N}$ a lei no espaço $D\left([0, T],\{0,1\}^{\boldsymbol{Z}}\right)$ do processo de exclusão $\left\{\eta_{t}: t \geq 0\right\}$ com distribuição inicial $\mu$ e gerador $L$ dado na equação (1.1) acelerado por $N^{2}$. A esperança com respeito a $\mathbb{P}_{\mu}^{\beta, N}$ será denotada por $\mathbb{E}_{\mu}^{\beta, N}$. 
Teorema 2 Seja $\rho_{0}: \mathbb{R} \rightarrow[0,1]$ uma função uniformemente contínua e seja $\left\{\mu_{N}: N \geq 1\right\}$ uma família de medidas de probabilidade em $\{0,1\}^{\boldsymbol{Z}}$ associadas a $\rho_{0}$. Isto é,

$$
\lim _{N \rightarrow \infty} \mu_{N}\left\{\left|\left\langle\pi_{0}^{N}, H\right\rangle-\int_{\mathbb{R}} H(u) \rho_{0}(u) d u\right|>\delta\right\}=0,
$$

para toda $H \in C_{c}(\mathbb{R})$ e todo $\delta>0$. Então, para todo $T>0, \delta>0$ e todo $H \in C_{c}(\mathbb{R})$,

$$
\lim _{N \rightarrow \infty} \int \mathbb{P}_{\mu}^{\beta, N}\left[\sup _{0 \leq t \leq T}\left|\left\langle\pi_{t}^{N}, H\right\rangle-\int_{\mathbb{R}} H(u)\left(T_{N}^{\beta}(t) \rho_{0}\right)(\lceil u N\rceil / N) d u\right|>\delta\right] \mathcal{D}(d \beta)=0,
$$

onde $T_{N}^{\beta}(t)$ é o semigrupo de Markov associado ao passeio aleatório $X_{N}^{\beta}(t \mid) /$.$N e (A, \Delta, \mathcal{D})$ é o espaço de probabilidade onde estão definidas as taxas $\beta_{x}, x \in \mathbb{Z}$.

\section{Demonstração}

Seja $K$ um subconjunto compacto de $\mathbb{R}$ que contém o suporte de $H$. Da definição de semigrupo temos que

$$
\sup _{0 \leq t \leq T}\left|\int_{\mathbb{R}} H(u)\left\{T(t) \rho_{0}(u)-T_{N}(t) \rho_{0}(u)\right\} d u\right|
$$

é limitado superiormente por

$$
C(H) \sup _{0 \leq t \leq T} \int_{K} \mathbb{E}\left[\left|\rho_{0}(Y(t, u))-\rho_{0}\left(Y_{N}(t, u)\right)\right|\right] d u,
$$

onde $C(H)$ é uma função que depende só de $H$.

Como $\rho_{0}$ é uniformemente contínua, existe $\delta>0$ para o qual a equação acima é menor ou igual a

$$
C(H) \epsilon+C\left(H, \rho_{0}\right) \int_{K} \mathbb{P}\left[\sup _{0 \leq t \leq T}\left|Y(t, u)-Y_{N}(t, u)\right|>\delta\right] d u
$$

onde $C\left(H, \rho_{0}\right)$ é uma função que depende de $H$ e do perfil inicial.

Dos resultados obtidos para os processos $Y(t, u)$ e $Y_{N}(t, u)$ e do Teorema da Convergência Dominada temos que

$$
\lim _{N \rightarrow \infty} C\left(H, \rho_{0}\right) \int_{K} \mathbb{P}\left[\sup _{0 \leq t \leq T}\left|Y(t, u)-Y_{N}(t, u)\right|>\delta\right] d u=0
$$


para todo $\delta>0$.

Portanto,

$$
\lim _{N \rightarrow \infty} \sup _{0 \leq t \leq T}\left|\int_{\mathbb{R}} H(u)\left\{T(t) \rho_{0}(u)-T_{N}(t) \rho_{0}(u)\right\} d u\right|=0
$$

Da equação (2.35) e do Teorema 1 segue que

$$
\lim _{N \rightarrow \infty} \mathbb{P}_{\mu_{N}}^{W, N}\left[\sup _{0 \leq t \leq T}\left|\left\langle\pi_{t}^{N}, H\right\rangle-\int_{\mathbb{R}} H(u)\left(T_{N}(t) \rho_{0}\right)(u) d u\right|>\delta\right]=0 .
$$

Como, para cada $N \geq 1,\left\{c_{x, N}: x \in \mathbb{Z}\right\}$ tem a mesma distribuição que $\left\{\beta_{x}: x \in \mathbb{Z}\right\}$, temos que

$$
\begin{aligned}
& \mathbb{P}_{\mu_{N}}^{W, N}\left[\sup _{0 \leq t \leq T}\left|\left\langle\pi_{t}^{N}, H\right\rangle-\int_{\mathbb{R}} H(u)\left(T_{N}(t) \rho_{0}\right)(u) d u\right|>\delta\right] \\
= & \mathbb{P}_{\mu_{N}}^{\beta, N}\left[\sup _{0 \leq t \leq T}\left|\left\langle\pi_{t}^{N}, H\right\rangle-\int_{\mathbb{R}} H(u)\left(T_{N}^{\beta}(t) \rho_{0}\right)(u) d u\right|>\delta\right]
\end{aligned}
$$

em distribuição. 


\section{Capítulo 3}

\section{Conglomerados}

Vamos obter uma cota superior e uma inferior para a distribuição do que chamamos de tempo de escape da partícula na origem. Mais precisamente,fixe $j$ e considere um perfil inicial $C_{j}$ que dá probabilidades muito altas para as partículas estarem posicionadas nos sítios $-j,-j+1, \ldots, j-1, j$ , $j$ inteiro positivo, e probabilidades muito próximas de zero fora desses sítios, neste caso o tempo de escape da partícula na origem é o tempo no qual a partícula que está inicialmente na origem consegue se movimentar pela primeira vez. Considere o seguinte perfil de densidade inicial $\rho_{0}^{j}: \mathbb{R} \rightarrow[0,1]$, dado por

$$
\rho_{0}^{j}(u / N)=\left\{\begin{array}{lr}
\exp \left\{\frac{-1}{(u+j+1)(j+1-u)}\right\}+1-\exp \left\{\frac{-1}{(j+1)^{2}}\right\} & -j-1<u<j+1 \\
1-\exp \left\{\frac{-1}{(j+1)|u|}\right\} & \text { caso contrário }
\end{array}\right.
$$

para $j \geq 1$ um inteiro fixado.

Seja $\mu^{N}$ uma seqüência de medidas produto de Bernoulli tais que:

$$
\mu^{N}\{\eta ; \eta(x)=1\}=\rho_{0}^{j}(x / N) .
$$

Temos que o comportamento hidrodinâmico do processo de exclusão é descrito pela equação (2.11)

$$
\begin{cases}\partial_{t} \rho_{j} & =\Delta \rho_{j} \text { nos intervalos }\left(\gamma_{j}, \gamma_{j+1}\right) \\ \partial_{x} \rho_{j}\left(t, x_{j}+\right) & =\partial_{x} \rho_{j}\left(t, x_{j}-\right) \\ \partial_{x} \rho_{j}\left(t, x_{j}+\right) & =\lambda\left[\rho_{j}\left(t, x_{j}-\right)-\rho_{j}\left(t, x_{j}+\right)\right] \\ \rho_{j}(0, .) & =\rho_{0}^{j}(.),\end{cases}
$$

onde $\rho_{j}(t, u)=T(t) \rho_{0}^{j}(u)$. 
Para uma realização de $W$, seja $\rho_{j, t}^{N, W}(x)=\mathbb{E}_{\mu^{N}}\left[\eta_{t}(x)\right]$. Como anteriormente, para simplificar a notação omitiremos a dependência de $W$ em $\rho_{j, t}^{N, W}(x)$ e escreveremos $\rho_{j, t}^{N}(x)$.

$\rho_{j, t}^{N}: \mathbb{Z} \rightarrow[0,1]$ é a solução da equação linear discreta dada por:

$$
\left\{\begin{array}{l}
\partial_{t} \rho_{t}^{N}(x)=N\left\{c_{x} \nabla_{N} \rho_{t}^{N}(x)-c_{x-1} \nabla_{N} \rho_{t}^{N}(x-1)\right\} \\
\rho_{0}^{N}(x)=\rho_{0}^{j}(x / N)
\end{array}\right.
$$

onde para $h: \mathbb{R} \rightarrow \mathbb{R},\left(\nabla_{N} h\right)(x)=N[h(x+1)-h(x)]$ e $c_{x}$ a função definida na equação $(2.2)$.

Resultado 7 ([5]) Fixe um perfil $u_{0}: \mathbb{R} \rightarrow \mathbb{R}$ com a quarta derivada limitada. Seja $u: \mathbb{R}_{+} \times \mathbb{R} \rightarrow$ $\mathbb{R}$ a solução da equação do calor com perfil inicial $u_{0}$,

$$
\left\{\begin{array}{l}
\partial_{t} u(t, x)=\partial_{x}^{2} u(t, x) \\
u(0, x)=u_{0}(x)
\end{array}\right.
$$

Para cada $N \in \mathbb{I}$, defina $u_{t}^{N}(x)$ como a solução do sistema de equações diferenciais ordinárias

$$
\begin{cases}(d / d t) u_{t}^{N}(x) & =\left(\Delta_{N} u_{t}^{N}\right)(x) \\ u_{0}^{N}(x) & =u_{0}(x / N)\end{cases}
$$

onde $\Delta_{N}$ representa o Laplaciano discreto. Então, existe uma constante finita $C_{0}>0$ tal que

$$
\left|u_{t}^{N}(x)-u(t, x / N)\right| \leq \frac{C_{0} t}{N^{2}}
$$

para todo $N \geq 1, t \geq 0, x \in \mathbb{Z}$

Em [5], esse resultado é colocado como uma aproximação da equação do calor por soluções da equação de calor discreta. Este resultado afirma que $u^{N}$ aproxima $u$ na ordem $N^{-2}$.

Vamos considerar uma aproximação a tempo discreto da equação (2.11).

Para cada $N \in \mathbb{I}, \delta>0$, defina $\rho_{l}^{\delta, N}(k), k \in \mathbb{Z}, l \geq 0$ pela seguinte fórmula de recorrência

$$
\begin{aligned}
\rho_{l+1}^{\delta, N}(k)= & \rho_{l}^{\delta, N}(k)+\delta N^{2}\left[c_{x, N} \rho_{l}^{\delta, N}(k+1)+c_{x-1, N} \rho_{l}^{\delta, N}(k-1)\right. \\
& \left.-\left(c_{x, N}+c_{x-1, N}\right) \rho_{l}^{\delta, N}(k)\right] \\
\rho_{0}^{\delta, N}(k)= & \rho_{0}(k / N)
\end{aligned}
$$


Portanto, se existir uma marca de Poisson entre os sítios $k$ e $k+1$, temos

$$
\begin{gathered}
\rho_{l+1}^{\delta, N}(k)=\rho_{l}^{\delta, N}(k)+\delta N^{2}\left[\frac{\lambda}{N} \rho_{l}^{\delta, N}(k+1)+\rho_{l}^{\delta, N}(k-1)-\left(1+\frac{\lambda}{N}\right) \rho_{l}^{\delta, N}(k)\right] \\
\rho_{l+1}^{\delta, N}(k+1)=\rho_{l}^{\delta, N}(k)+\delta N^{2}\left[\rho_{l}^{\delta, N}(k+1)+\frac{\lambda}{N} \rho_{l}^{\delta, N}(k-1)-\left(1+\frac{\lambda}{N}\right) \rho_{l}^{\delta, N}(k)\right] .
\end{gathered}
$$

Caso contrário, $c_{k, N}=c_{k-1, N}=c_{k+1, N}=1$ na Equação (3.4).

Proposição 1 Tome $\delta N^{2}<1 / 2$. Então existe uma constante finita $C\left(\rho_{0}\right)$ tal que

$$
\left|\rho_{l}^{\delta, N}(k)-\rho(\delta l, k / N)\right| \leq C\left(\rho_{0}\right) \delta l\left\{\delta+\frac{1}{N^{2}}\right\} \quad \forall l \geq 0
$$

onde $\rho(.,$.$) é a solução da equação de difusão com fronteiras (2.11).$

\section{Demonstração}

Vamos denotar por $k_{i}$ e $\left(k_{i}+1\right)$ aos sítios tais que a marca de Poisson $\gamma_{i}$ está entre eles. Primeiro vamos considerar os sítios pertencentes ao intervalo $\left(\left(k_{i-1}+1\right), k_{i}\right)$. Observe que o Resultado 7 para os sítios pertencentes a estes intervalos, permite afirmar que

$$
\left|\rho_{l}^{\delta, N}(k)-\rho(\delta l, k / N)\right| \leq C_{1}\left(\rho_{0}\right) \delta l\left\{\delta+\frac{1}{N^{2}}\right\} \quad \forall l \geq 0
$$

Vamos estudar o que acontece nos sítios $k_{i}$ e $(k+1)_{i}$.

Definimos os quocientes de diferenças para frente e para trás para todo $x \in \mathbb{Z}$ :

$$
\begin{aligned}
& \partial_{x} V_{t}^{N}(x)=N\left(V_{t}^{N}(x+1)-V_{t}^{N}(x)\right) \\
& \bar{\partial}_{x} V_{t}^{N}(x)=N\left(V_{t}^{N}(x)-V_{t}^{N}(x-1)\right)
\end{aligned}
$$

Assim a equação de diferenças pode ser escrita como

$$
\partial_{x}\left[\bar{\partial}_{x} V_{t}^{N}(x)\right]=N^{2}\left[V_{t}^{N}(x+1)+V_{t}^{N}(x-1)-2 V_{t}^{N}(x)\right] .
$$


Segue que,

$$
\begin{aligned}
\partial_{t} \rho_{l}^{\delta, N}\left(k_{i}\right) & =N^{2}\left[\frac{\lambda}{N} \rho_{l}^{\delta, N}\left(k_{i}+1\right)+\rho_{l}^{\delta, N}\left(k_{i}-1\right)-\left(1+\frac{\lambda}{N}\right) \rho_{l}^{\delta, N}\left(k_{i}\right)\right] \\
& =\partial_{x}\left[\bar{\partial}_{x} \rho_{l}^{\delta, N}\left(k_{i}\right)\right]+N^{2}\left(\frac{\lambda}{N}-1\right)\left[\rho_{l}^{\delta, N}\left((k+1)_{i}\right)-\rho_{l}^{\delta, N}\left(k_{i}\right)\right] .
\end{aligned}
$$

Vamos considerar o operador $\mathbf{E}_{\delta N^{2}}$ tal que, para todo $x \in \mathbb{Z}$,

$$
\mathbf{E}_{\delta N^{2}} \rho_{l}^{\delta, N}(x)=\rho_{l}^{\delta, N}(x)+\delta N^{2}\left[\rho_{l}^{\delta, N}(x+1)+\rho_{l}^{\delta, N}(x-1)-2 \rho_{l}^{\delta, N}(x)\right]
$$

Quando $\delta N^{2}<1 / 2$ os coeficientes do operador $\mathbf{E}_{\delta N^{2}}$ são todos positivos e a soma deles é um. Logo, pela definição do operador $\mathbf{E}_{\delta N^{2}}$ e de $\rho_{l}^{\delta, N}$ nos sítios $k_{i}$ e $(k+1)_{i}$, temos que

$$
\begin{gathered}
\rho_{l+1}^{\delta, N}\left(k_{i}\right)=\mathbf{E}_{\delta N^{2}} \rho_{l}^{\delta, N}\left(k_{i}\right)+\delta N^{2}\left(\frac{\lambda}{N}-1\right)\left[\rho_{l}^{\delta, N}\left((k+1)_{i}\right)-\rho_{l}^{\delta, N}\left(k_{i}\right)\right], \\
\rho_{l+1}^{\delta, N}\left((k+1)_{i}\right)=\mathbf{E}_{\delta N^{2}} \rho_{l}^{\delta, N}\left((k+1)_{i}\right)+\delta N^{2}\left(\frac{\lambda}{N}-1\right)\left[\rho_{l}^{\delta, N}\left(k_{i}\right)-\rho_{l}^{\delta, N}\left((k+1)_{i}\right)\right] .
\end{gathered}
$$

Por outro lado, para todo $x \in\left(\gamma_{i-1}, \gamma_{i}\right) \bigcup\left(\gamma_{i}, \gamma_{i+1}\right), \rho(t, x)$ satisfaz a equação do calor

$$
\left\{\begin{array}{l}
\partial_{t} \rho(t, x)=\partial_{x}^{2} \rho(t, x) \\
\rho(0, x)=\rho_{0}(x)
\end{array}\right.
$$

Portanto, temos, por expansão de Taylor, que para $y \in\left\{k_{i},(k+1)_{i}\right\}$,

$$
\begin{aligned}
\rho(\delta(t+1), y / N) & =\rho(\delta t, y / N)+\delta\left(\frac{\partial}{\partial t} \rho(\delta t, y / N)\right)+O\left(\delta^{2}\right) \\
& =\rho(\delta t, y / N)+\delta\left(\frac{\partial^{2}}{\partial x^{2}} \rho(\delta t, y / N)\right)+O\left(\delta^{2}\right) \\
& =\rho(\delta t, y / N)+\delta\left(\partial_{x} \bar{\partial}_{x} \rho(\delta t, y / N)+O\left(N^{-2}\right)\right)+O\left(\delta^{2}\right)
\end{aligned}
$$

Segue que,

$$
\partial_{t} \rho(\delta t, y / N)-\partial_{x} \bar{\partial}_{x} \rho(\delta t, y / N)=O\left(N^{-2}+\delta\right)
$$

Agora, para $y \in\left\{k_{i},(k+1)_{i}\right\}$, seja $z(\delta l, y / N)=\rho_{l}^{\delta, N}(y)-\rho(\delta l, y / N)$. Logo, das equações (3.8),(3.6) e (3.7), segue que 


$$
\partial_{t} z\left(\delta l, k_{i} / N\right)-\partial_{x} \bar{\partial}_{x} z\left(\delta l, k_{i} / N\right)=N^{2}\left(\frac{\lambda}{N}-1\right)\left[\rho_{l}^{\delta, N}\left((k+1)_{i}\right)-\rho_{l}^{\delta, N}\left(k_{i}\right)\right]-O\left(N^{-2}+\delta\right)
$$

Além disso,

$$
\begin{aligned}
z\left(\delta(l+1), k_{i} / N\right)= & \mathbf{E}_{\delta N^{2}} z\left(\delta(l+1), k_{i} / N\right)+\delta N^{2}\left(\frac{\lambda}{N}-1\right)\left[\rho_{l}^{\delta, N}\left((k+1)_{i}\right)-\rho_{l}^{\delta, N}\left(k_{i}\right)\right] \\
& -\delta O\left(N^{-2}+\delta\right) \\
= & \mathbf{E}_{\delta N^{2}}^{l+1} z\left(0, k_{i} / N\right)-\delta \sum_{j=0}^{l} \mathbf{E}_{\delta N^{2}}^{l-j} O\left(N^{-2}+\delta\right)+ \\
& \delta N^{2}\left(\frac{\lambda}{N}-1\right) \sum_{j=0}^{l} \mathbf{E}_{\delta N^{2}}^{l-j}\left[\rho_{j}^{\delta, N}\left((k+1)_{i}\right)-\rho_{j}^{\delta, N}\left(k_{i}\right)\right] .
\end{aligned}
$$

\section{Portanto,}

$$
\begin{aligned}
\left|z\left(\delta(l), k_{i} / N\right)\right| \leq & \delta \sum_{j=0}^{l-1}\left|O\left(N^{-2}+\delta\right)\right|+\delta N^{2}\left(\frac{\lambda}{N}-1\right) \times \\
& \left|\sum_{j=0}^{l-1} \mathbf{E}_{\delta N^{2}}^{l-j-1}\left[\rho_{j}^{\delta, N}\left((k+1)_{i}\right)-\rho_{j}^{\delta, N}\left(k_{i}\right)\right]\right| \\
\leq & C_{2}\left(\rho_{0}\right) \delta l\left(\delta+\frac{1}{N^{2}}\right)+\delta N^{2}\left(\frac{\lambda}{N}-1\right) \times \\
& \left|\sum_{j=0}^{l-1} \mathbf{E}_{\delta N^{2}}^{l-j-1}\left[\rho_{j}^{\delta, N}\left((k+1)_{i}\right)-\rho_{j}^{\delta, N}\left(k_{i}\right)\right]\right|
\end{aligned}
$$

e, com o mesmo argumento usado para $y=(k+1)_{i}$, segue que

$$
\begin{gathered}
\left|z\left(\delta(l),(k+1)_{i} / N\right)\right| \leq C_{2}\left(\rho_{0}\right) \delta l\left(\delta+\frac{1}{N^{2}}\right)+\delta N^{2}\left(\frac{\lambda}{N}-1\right) \\
\times\left|\sum_{j=0}^{l-1} \mathbf{E}_{\delta N^{2}}^{l-j-1}\left[\rho_{j}^{\delta, N}\left(k_{i}\right)-\rho_{j}^{\delta, N}\left((k+1)_{i}\right)\right]\right| .
\end{gathered}
$$

Finalmente, para $y \in\left\{k_{i},(k+1)_{i}\right\}$, 


$$
\begin{aligned}
|z(\delta(l), y / N)| & \leq\left|z\left(\delta(l), k_{i} / N\right)\right|+\left|z\left(\delta(l),(k+1)_{i} / N\right)\right| \\
& \leq C_{3}\left(\rho_{0}\right) \delta l\left(\delta+\frac{1}{N^{2}}\right) \quad \forall l \geq 0 .
\end{aligned}
$$

Da Proposição 1 segue que para $N \geq 1$,

$$
\lim _{\delta \rightarrow 0} \rho_{\lfloor t / \delta\rfloor}^{\delta, N}(k)=\rho_{t}^{N}(k) .
$$

Do limite acima, da Proposição 1 e do Resultado 7 segue que para $\rho_{0}: \mathbb{R} \rightarrow[0,1]$ um perfil com a quarta derivada limitada; existe uma constante finita $C_{0}>0$ tal que

$$
\left|\rho_{t}^{N}(x)-\rho(t, x / N)\right| \leq \frac{C_{0} t}{N^{2}}
$$

para todo $N \geq 1, t \geq 0, x \in \mathbb{Z}$.

Do resultado acima segue que $\rho_{j}^{N}$ aproxima $\rho_{j}$ na ordem $N^{-2}$. Isto é

$$
\left|\rho_{j, t}^{N}(x)-\rho_{j}(t, x / N)\right| \leq \frac{C_{0} t}{N^{2}}
$$

para todo $N \geq 1, t \geq 0, j \geq 1, x \in \mathbb{Z}, C_{0}>0$ finita.

Para $x \in \mathbb{Z}$ e $t \geq 0$ defina $\alpha_{t}^{x}=\lambda t / N$ se existir uma marca de Poisson entre $(x, x+1)$ e $\alpha_{t}^{x}=t$ caso contrário e seja $b(i, k, a, s)=\sum_{i=\{1,-1\}} \int_{a}^{t} \rho_{j}(s, i / N) d \alpha_{s}^{k}$.

Proposição 2 Fixe $j \in \mathbb{N}$. Considere o perfil inicial $\rho_{0}^{j}$. Denote por $T_{j}$ o tempo em que a origem fica vazía pela primeira vez. Segue que para todo $t \geq 0$

$$
\mathbb{P}\left\{T_{j} \geq t\right\} \geq \rho_{0}^{j}(0)-\sum_{i=\{1,-1\}} \int_{0}^{t}\left(1+\frac{C_{0} s}{N^{2}}\right) d \alpha_{s}^{i}+b(i, i, 0, s)
$$


Proposição 3 Fixe $j \in \mathbb{I N}$. Considere o perfil inicial $\rho_{0}^{j}$. Seja

$$
A(b(i, k, a, s))=1-\rho_{0}^{j}(0)+\sum_{i=\{1,-1\}} \int_{0}^{t}\left(1+\frac{C_{0} s}{N^{2}}\right) d \alpha_{s}^{i}-b(i, k, a, s) .
$$

Para todo $t \geq 0$,

$$
\mathbb{P}\left\{T_{j} \geq t\right\} \leq 1-\frac{(A(b(i, i, 0, s)))^{2}}{A(b(i, i, 0, s))+2 \int_{0}^{t} \int_{s}^{t} \sum_{i, k=\{1,-1\}}\left(1+\frac{C_{0} u}{N^{2}}\right) d \alpha_{u}^{i} d \alpha_{s}^{k}-2 \int_{0}^{t} \sum_{i=\{1,-1\}} b(k, i, s, u) d \alpha_{s}^{k}}
$$

\subsection{Demonstrações das proposições}

\section{Demonstração da Proposição 2}

Definimos processos independentes de Poisson $\left\{\tau_{k}^{(x, y)}: k \geq 0\right\} \operatorname{nos}$ elos $(x, y)$ onde $\left\{\tau_{k}^{(x, y)}\right\}$ são instantes aleatórios nos quais a partícula tenta-se movimentar de $x$ a $y=x+1$ com taxa $c_{x}$ e de $x$ a $y=x-1$ com taxa $c_{x-1}$.

Para $y, x \in \mathbb{Z}$ tal que $|x-y|=1$, seja

$$
N_{t}^{\{x, y\}}=\sum_{k \geq 1} \mathbf{I}_{\left\{\tau_{k}^{(x, y)} \leq t\right\}}
$$

para $t>0$ e $N_{0}^{\{x, y\}}=0 . \operatorname{Logo}, N_{t}^{\{x, y\}}$ conta o número de ocorrências de $\left\{\tau_{k}^{(x, y)}\right\}$ no par $\{x, y\}$ em $[0, t]$. Assim, se não existir nenhuma marca de Poisson $\gamma_{n}$ entre $(x, x+1), N_{t}^{\{x, x+1\}}$ é um processo de Poisson de parâmetro (intensidade) 1. Portanto, $N_{t}^{\{x, y\}}$ tem uma distribuição de Poisson com parâmetro $t$. Por outro lado, se existir ao menos uma marca de Poisson entre $(x, x+1)$;o processo $N_{t}^{\{x, x+1\}}$ é um processo de Poisson com parâmetro $\frac{\lambda t}{N}$. 
Seja $\left\{\tau_{n}\right\}$ a superposição dos processos de Poisson $\tau_{k}^{\{0,1\}}$ e $\tau_{k}^{\{0,-1\}}$. Defina o processo $Y^{C_{j}}[s, t]$ como

$$
Y^{C_{j}}[s, t]=\mathbf{I}_{\left\{\eta_{s}^{C_{j}}(0)=0\right\}}+\sum_{k=1}^{\infty} \mathbf{I}_{\left\{s<\tau_{k}<t ; \eta_{\tau_{k}}\right.}^{\left.C_{j}(0)=0\right\}}
$$

Temos que $Y^{C_{j}}[s, t]$ conta o número de vezes que a origem ficou desocupada no intervalo $[s, t]$.

Definimos as seguintes funções para $i=1,-1$.

$$
\phi_{t}^{i, C_{j}}=\mathbf{I}_{\left\{\eta_{t}^{C_{j}}(i)=0\right\}}
$$

Seja $V=\{1,-1\}$ Segue que,

$$
Y^{C_{j}}[0, t]=\mathbf{I}_{\left\{\eta^{C_{j}}(0)=0\right\}}+\sum_{i \in V} \int_{0}^{t} \phi_{s^{-}}^{i, C_{j}} d N_{s}^{\{i, 0\}}
$$

onde $\phi_{t^{-}}=\lim _{r \uparrow t} \phi_{r}$.

Observe que $N_{t}^{\{x, y\}}-\alpha_{t}^{x}$ é martingal de média zero, e para cada $i \in V$ os $\phi_{t^{-}}^{i, C_{j}}$ são previsíveis respeito à filtração $\mathcal{F}(-\infty, t-)$.

Temos que,

$$
\sum_{i \in V} \int_{0}^{t} \phi_{s^{-}}^{i, C_{j}} d N_{s}^{\{i, 0\}}=\sum_{i \in V} \int_{0}^{t} \phi_{s^{-}}^{i, C_{j}} d\left(N_{s}^{\{i, 0\}}-\alpha_{s}^{i}\right)+\sum_{i \in V} \int_{0}^{t} \phi_{s^{-}}^{i, C_{j}} d \alpha_{s}^{i}
$$


e segue que

$$
\begin{aligned}
\mathbb{E} Y^{C_{j}}[0, t] & =\mathbb{P}\left\{\eta^{C_{j}}(0)=0\right\}+\sum_{i \in V} \int_{0}^{t} \mathbb{E} \phi_{s^{-}}^{i, C_{j}} d \alpha_{s}^{i} \\
& =1-\rho_{0}^{j}(0)+\sum_{i \in V} \int_{0}^{t} \mathbb{P}\left(\eta_{s^{-}}^{C_{j}}(i)=0\right) d \alpha_{s}^{i} \\
& =1-\rho_{0}^{j}(0)+\sum_{i \in V} \int_{0}^{t}\left(1-\rho_{j, s^{-}}^{N}(i)\right) d \alpha_{s}^{i} \\
& \leq 1-\rho_{0}^{j}(0)+\sum_{i \in V} \int_{0}^{t}\left(1+\frac{C_{0} s}{N^{2}}\right) d \alpha_{s}^{i}-b(i, i, 0, s)
\end{aligned}
$$

onde a última desigualdade se deve à equação (3.12). Pela desigualdade de Markov temos que $\mathbb{P}\left(Y^{C_{j}}[0, t] \geq 1\right) \leq \mathbb{E} Y^{C_{j}}[0, t]$. Segue que

$$
\mathbb{P}\left\{T_{j} \leq t\right\}=\mathbb{P}\left(Y^{C_{j}}[0, t] \geq 1\right) \leq \mathbb{E} Y^{C_{j}}[0, t] .
$$

Logo,

$$
\mathbb{P}\left\{T_{j} \geq t\right\} \geq \rho_{0}^{j}(0)-\sum_{i=\{1,-1\}} \int_{0}^{t}\left(1+\frac{C_{0} s}{N^{2}}\right) d \alpha_{s}^{i}+b(i, i, 0, s) .
$$

\section{Demonstração da Proposição 3}

Observe que,

$$
\mathbb{P}[Y \geq 1] \geq \frac{(\mathbb{E} Y)^{2}}{\mathbb{E}\left(Y^{2}\right)} \quad \text { onde } Y=Y[0, t]
$$

pois pela desigualdade de Cauchy-Schwars segue que

$$
(\mathbb{E} Y)^{2}=\left[\mathbb{E}\left(Y . \mathbf{I}_{\{Y \geq 1\}}\right)\right]^{2} \leq \mathbb{E}\left(Y^{2}\right) \mathbb{P}(Y \geq 1) .
$$

Como $Y$ é uma soma de funções indicadoras, segue que

$$
\left(Y^{C_{j}}\right)^{2}=Y^{C_{j}}+2 \int_{0<s<u<t} \sum_{x, y \in\{1,-1\}} \phi_{s^{-}}^{x} \phi_{u^{-}}^{y} d N_{u}^{x, x+1} d N_{s}^{y, y+1} .
$$


Temos que

$\mathbb{E} \underset{0<s<u<t}{\int_{s^{-}}^{x}} \phi_{u^{-}}^{y} d N_{u}^{x, x+1} d N_{s}^{y, y+1}$ é igual a

$$
\mathbb{E} \int_{0<s<u<r} \phi_{s^{-}}^{x} \phi_{u^{-}}^{y}\left(d N_{u}^{x, x+1}-d \alpha_{u}^{x}\right) d N_{s}^{y, y+1}+\mathbb{E} \int_{0<s<u<r} \phi_{s^{-}}^{x} \phi_{u^{-}}^{y} d \alpha_{u}^{x} d N_{s}^{y, y+1}
$$

Como $\mathbf{I}_{\left\{\eta_{s^{-}}^{C_{j}}(x)=0\right\}} \times \mathbf{I}_{\left\{\eta_{u^{-}}^{C_{j}}(y)=0\right\}}$ é uma função previsível respeito à filtração $\left(\mathcal{F}\left(-\infty, u^{-}\right)\right) \mathrm{e}$ $\left(N_{u}^{x, x+1}-\alpha_{u}^{x}\right)$ é martingal de média zero, segue que

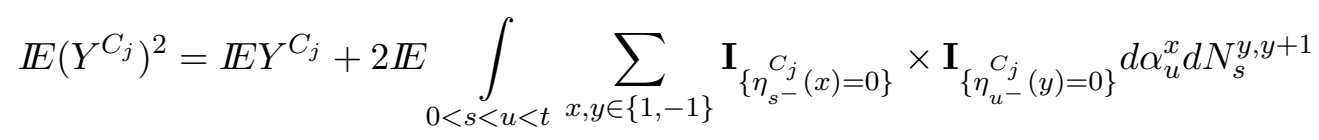

Seja $B(\eta, s)=\mathbb{E}\left[\int_{s}^{t} \mathbf{I}_{\left\{\eta_{u^{-}}{ }^{C_{j}}(y)=0\right\}} d \alpha_{u}^{x} \mid \eta_{s}=\eta\right]$. Como $B\left(\eta_{s}, s\right) \mathbf{I}_{\left\{\eta_{s^{-}}{ }^{C_{j}}(x)=0\right\}}$ é $\left(\mathcal{F}\left(-\infty, s^{-}\right)\right)$mensurável, e $\left(N_{s}^{y, y+1}-\alpha_{s}^{y}\right)$ é martingal. Segue que,

$$
\begin{aligned}
& \mathbb{E} \int_{0<s<u<t} \mathbf{I}_{\left\{\eta_{s^{-}} C_{j}(x)=0\right\}} \times \mathbf{I}_{\left\{\eta_{u^{-}} C_{j}(y)=0\right\}} d \alpha_{u}^{x} d N_{s}^{y, y+1}=\mathbb{E} \int_{0<s<u<t} \mathbf{I}_{\left\{\eta_{s^{-}} C^{C_{j}}(x)=0\right\}} B\left(\eta_{s}, s\right) d N_{s}^{y, y+1} \\
&=\mathbb{E} \int_{0<s<u<t} \mathbf{I}_{\left\{\eta_{s^{-}} C_{j}(x)=0\right\}} B\left(\eta_{s}, s\right) d \alpha_{s}^{y} \\
&=\mathbb{E} \int_{0}^{t} \int_{s}^{t} \mathbf{I}_{\left\{\eta_{s^{-}} C_{j}(x)=0\right\}} \mathbf{I}_{\left\{\eta_{u}{ }^{C}(y)=0\right\}} d \alpha_{u}^{x} d \alpha_{s}^{y}
\end{aligned}
$$

Portanto,

$$
\mathbb{E} Y^{2}=\mathbb{E} Y+2 \mathbb{E} \sum_{x, y \in\{1,-1\}} \int_{0}^{t} \int_{s}^{t} \mathbf{I}_{\left\{\eta_{s^{-}} C_{j}(x)=0\right\}} \mathbf{I}_{\left\{\eta_{u^{-}} C^{C_{j}}(y)=0\right\}} d \alpha_{u}^{x} d \alpha_{s}^{y}
$$

Além disso, 


$$
\begin{aligned}
\mathbb{E}\left[\mathbf{I}_{\left\{\eta_{s^{-}} C_{j}(x)=0\right\}} \mathbf{I}_{\left\{\eta_{u^{-}} C_{j}(y)=0\right\}}\right] & =\mathbb{P}\left(\eta_{s^{-}}(x)=0, \eta_{u^{-}}(y)=0\right) \\
& =1-\mathbb{P}\left(\eta_{u^{-}}(y)=1\right)-\mathbb{P}\left(\eta_{s^{-}}(x)=1, \eta_{u^{-}}(y)=0\right) \\
& \leq 1-\mathbb{P}\left(\eta_{u^{-}}(y)=1\right)=1-\rho_{j, u^{-}}^{N}(y) .
\end{aligned}
$$

Portanto, das equações (3.17) e (3.18) segue que

$$
\begin{aligned}
\mathbb{E} Y^{2} & \leq \mathbb{E} Y+2 \int_{0}^{t} \int_{s}^{t} \sum_{x, y \in\{1,-1\}}\left(1-\rho_{j, u}^{N}(y)\right) d \alpha_{u}^{x} d \alpha_{s}^{y} \\
& =\mathbb{E} Y+2 \int_{0}^{t} \sum_{x \in V} \int_{s}^{t} \sum_{y \in V}\left(1-\rho_{j, u}^{N}(y)\right) d \alpha_{u}^{x} d \alpha_{s}^{y} \\
& \leq \mathbb{E} Y+2 \int_{0}^{t} \sum_{x \in V} \int_{s}^{t}\left(1+\frac{C_{0} u}{N^{2}}-\rho_{j}(u, y / N)\right) d \alpha_{u}^{x} d \alpha_{s}^{y} \\
& =\mathbb{E} Y+2 \int_{0}^{t} \int_{s}^{t} \sum_{x, y=\{1,-1\}}\left(1+\frac{C_{0} u}{N^{2}}\right) d \alpha_{u}^{x} d \alpha_{s}^{y}-2 \int_{0}^{t} \sum_{i=\{1,-1\}} b(y, x, s, u) d \alpha_{s}^{y}
\end{aligned}
$$

onde na segunda desigualdade acima, usou-se o resultado (3.12).

Portanto, substituindo a desigualdade (3.13) em (3.19) segue que

$$
\begin{aligned}
\mathbb{E} Y^{2} \leq 1-\rho_{0}^{j}(0) & +\sum_{i=\{1,-1\}} \int_{0}^{t}\left(1+\frac{C_{0} s}{N^{2}}\right) d \alpha_{s}^{i}-b(i, i, 0, s)+ \\
& 2 \int_{0}^{t} \int_{s}^{t} \sum_{x, y=\{1,-1\}}\left(1+\frac{C_{0} u}{N^{2}}\right) d \alpha_{u}^{x} d \alpha_{s}^{y}-2 \int_{0}^{t} \sum_{i=\{1,-1\}} b(y, x, s, u) d \alpha_{s}^{y}
\end{aligned}
$$

Substituindo as equações (3.20) e (3.13) na equação (3.14), temos que uma cota superior de $\mathbb{P}\left\{T_{j} \geq t\right\}$ é dada por

$$
1-\frac{(A(b(i, i, 0, s)))^{2}}{A(b(i, i, 0, s))+2 \int_{0}^{t} \int_{s}^{t} \sum_{x, y=\{1,-1\}}\left(1+\frac{C_{0} u}{N^{2}}\right) d \alpha_{u}^{x} d \alpha_{s}^{y}-2 \int_{0}^{t} \sum_{x=\{1,-1\}} b(y, x, s, u) d \alpha_{s}^{y}} .
$$




\section{Bibliografia}

[1] Timo Seppäläinen (2005) Translation Invariant Exclusion Processes Department of Mathematics, University of Winconsin.

[2] P.A Ferrari, A. Galves, T.Liggett (1995) Exponential waiting time for filling a large interval in the symmetric single exclusion process.Ann. Inst. H. Poincaré. Probabilités et Statistique 31 (1) $155-175$.

[3] P.A Ferrari, A. Galves, C. Landim (2000) Rate of convergence to equilibrium of symmetric simple exlcusion processes.Markov Processes and Related Fields 6 (2000), 73-88

[4] Frank Spitzer (1926) Principles of Random Walk.Springer -Verlag Berlin

[5] M.D.Jara, C. Landim (2006) Nonequilibrium central limit theorem for a tagged particle in symmetric simple exclusion. Annales de l'Institut Henri Poincare. B, Probability and Statistics, v. 42, p. A, 2006.

[6] Gregory Lawler F. (1991) Intersections of Random Walks Boston: Birkhäuser.

[7] C.Kipnic, C Landim (1999) Scaling Limits of Interacting Particle Systems Germany:Springer [8] Adilson Simonis (1995) Metastability of the $d$-dimensional contact process Journal of Statistical Physics, Vol. 83. Nos. 5/6. 1996.

[9] Révész Pál (1990) Random Walk in random and non-random enviroments Singapore: Teareck, NJ, World Scientifics.

[10] Thomas Liggett (1985) Interacting Particle Systems New York: Springer. Verlang

[11] P.A Ferrari, A. Galves (1997) Acoplamento e Processos estocásticos. IMPA. $21^{\circ}$ Coloquio Brasileiro de matemática.

[12] Norman L. Johnson, Samuel Kotz, N. Balakrishnan (1970) Continuous Univariate distributions. Volume 2. Second edition

[13] Norman L. Johnson, Samuel Kotz, N. Balakrishnan (1994) Continuous Univariate distributions. Volume 1. Second edition.New York. Chichester.

[14] Vladimir Belitsky (1993) Asymptotic Upper Bound of Density for Two-Particle Annihilating Exclusion. The Journal of Statistical Physics, Vol. 73, N. 3/4, pp.671-694. 
[15]A.Faggionato,M.Jara,C.Landim (2007) Hydrodinamic limit of one dimensional subdiffusive exclusion processes with random conductance.

[16]Charles Stone(1963) Limit theorems for random walks, birth and death processes, and diffusion processes I11.J.Math 7,638-660.

[17] Petr Mandl (1968) Analytical Tratment of One-dimensional Markov Processes. Springer Verlang Beriln Heidelberg.

[18] K.Nagy (2002) Symmetric random walk in random environment. Period.Math.Hung.45, 101120 .

[19] C.Landim, S.Olla, S.B.Volchan (1998)Driven Tracer Particle in One Dimensional Symmetric Simple Exclusion. Commun.Math.Phys.192,287-307 . 